\title{
Exploring the new physics phases in $3+1$ scenario in neutrino oscillation experiments
}

\author{
Nishat Fiza, ${ }^{a}$ Mehedi Masud ${ }^{b, c}$ and Manimala Mitra ${ }^{c, d}$ \\ ${ }^{a}$ Department of Physical Sciences, IISER Mohali, Knowledge City, \\ SAS Nagar, Mohali 140306, Punjab, India \\ ${ }^{b}$ Center for Theoretical Physics of the Universe (CTPU), Institute for Basic Science (IBS), \\ 55 Expo-ro, Daejeon 34126, Korea \\ ${ }^{c}$ Institute of Physics, \\ Sachivalaya Marg, Sainik School Post, Bhubaneswar 751005, Odisha, India \\ ${ }^{d}$ Homi Bhabha National Institute, BARC Training School Complex, \\ Anushakti Nagar, Mumbai 400094, India \\ E-mail: ph15039@iisermohali.ac.in, masud@ibs.re.kr, \\ manimala@iopb.res.in
}

ABSTRACT: The various global analyses of available neutrino oscillation data indicate the presence of the standard $3+0$ neutrino oscillation picture. However, there are a few short baseline anomalies that point to the possible existence of a fourth neutrino (with mass in the eV-scale), essentially sterile in nature. Should sterile neutrino exist in nature and its presence is not taken into consideration properly in the analyses of neutrino data, the interference terms arising due to the additional $\mathrm{CP}$ phases in presence of a sterile neutrino can severely impact the physics searches in long baseline (LBL) neutrino oscillation experiments. In the current work we consider one light (eV-scale) sterile neutrino and probe all the three $\mathrm{CP}$ phases $\left(\delta_{13}, \delta_{24}, \delta_{34}\right)$ in the context of the upcoming Deep Underground Neutrino Experiment (DUNE) and also estimate how the results improve when data from NOvA, T2K and T2HK are added in the analysis. We illustrate the $\Delta \chi^{2}$ correlations of the $\mathrm{CP}$ phases among each other, and also with the three active-sterile mixing angles. Finally, we briefly illustrate how the relevant parameter spaces in the context of neutrinoless double beta decay get modified in light of the bounds in presence of a light sterile neutrino.

Keywords: Beyond Standard Model, CP violation, Neutrino Physics

ArXiv EPrint: 2102.05063 


\section{Contents}

1 Introduction 1

2 Basics 4

$\begin{array}{lll}3 & \text { Simulation details } & 7\end{array}$

4 Reconstruction of the CP phase in correlation with other phases $\quad 9$

5 Role of individual channels in reconstruction 12

6 Reconstruction of the CP phase in correlation with active-sterile $\begin{array}{ll}\text { mixing angles } & 13\end{array}$

7 Impact of constraints on neutrinoless double beta decay 14

8 Summary and conclusion $\quad 16$

$\begin{array}{ll}\text { A Effect of } \theta_{23} \text {-uncertainty } & 17\end{array}$

B Effect of different true choice of the standard parameters 18

C Understanding the role of channels to probe $\delta_{24}$ and $\delta_{34} \quad 19$

\section{Introduction}

The successful discovery of the phenomena of neutrino oscillation $[1,2]$ has led to an impressive amount of research with an aim to establish the standard 3-flavour (referred to as $3+0$ hereafter) oscillation over a wide range of energy $(E)$ and neutrino propagation length $(L)$. In the commonly used Pontecorvo-Maki-Nakagawa-Sakata (PMNS) parametrization [3] of the leptonic mixing matrix, this requires fitting of the six standard oscillation parameters, namely three mixing angles $\left(\theta_{12}, \theta_{13}, \theta_{23}\right)$, two mass squared differences $\left(\Delta m_{21}^{2}=m_{2}^{2}-m_{1}^{2}, \Delta m_{31}^{2}=m_{3}^{2}-m_{1}^{2}\right)$ and one Dirac CP phase $\left(\delta_{13}\right)$. A complete knowledge about the oscillation parameters will help to shed light on the following still unresolved issues in the neutrino sector: whether there exists $\mathrm{CP}$ violation (CPV) in the leptonic sector (i.e., whether $\delta_{13} \neq 0, \pi$ ), whether the neutrino mass eigenstates are arranged in normal ordering (NO, i.e., $\Delta m_{31}^{2}>0$ ) or inverted ordering (IO, i.e., $\Delta m_{31}^{2}<0$ ), and, whether $\theta_{23}$ lies in the higher octant (HO, i.e., $\theta_{23}>\pi / 4$ ) or in the lower octant (LO, i.e., $\theta_{23}<\pi / 4$ ). If leptonic CPV exists, it would provide a crucial missing ingredient [4] in resolving another very fundamental elusive puzzle that is baryon asymmetry in the observed universe via a mechanism called Leptogenesis [5]. Determination of mass 
ordering and $\theta_{23}$ octant will help in understanding the origin of neutrino mass [6-8], its Dirac/Majorana nature via neutrinoless double beta decay [9] and in exploring a new symmetry called $\mu-\tau$ symmetry [10, 11]. Presently running long baseline (LBL) experiments such as Tokai to Kamioka (T2K) [12] and NuMI Off-axis $\nu_{e}$ Appearance (NO $\nu$ A) [13] have started uncovering a few of the open issues mentioned above. Latest T2K results [14] have been able to rule out a large range of values of $\delta_{13}$ around $\pi / 2$ at $3 \sigma$ confidence level (C.L.) irrespective of mass ordering. It also excludes CP conservation $(\delta=0$ or $\pi)$ at 95\% C.L. $\mathrm{NO} \nu \mathrm{A}$, in its latest dataset [15] using both $\nu$ and $\bar{\nu}$ running mode hint towards NO at $1.9 \sigma$ C.L. and shows a weak preference for $\theta_{23}$ lying in HO at a C.L. of 1.6 $\sigma$. Upcoming LBL experiments such as the Deep Underground Neutrino Experiment (DUNE) [16-18], Tokai to Hyper-Kamiokande (T2HK) [19], Tokai to Hyper-Kamiokande with a second detector in Korea (T2HKK) [20], European Spallation Source $\nu$ Super Beam (ESS $\nu$ SB) [21] as well as future data from $\mathrm{T} 2 \mathrm{~K}$ and $\mathrm{NO} \nu \mathrm{A}$ are expected to resolve the issues mentioned above with an unprecedented level of precision.

Various global analyses [22-25], analyzing neutrino data from a diverse array of sources such as atmosphere, particle accelerators, sun and nuclear reactors have consolidated the effort to build up the $3+0$ oscillation picture. There are however, a few short-baseline (SBL) anomalies [26-30] that hint towards the existence of oscillation governed by $\mathcal{O}\left(\mathrm{eV}^{2}\right)$ mass squared difference $\left(\Delta m_{41}^{2}=m_{4}^{2}-m_{1}^{2} \sim \mathrm{eV}^{2}\right)$ that cannot be accommodated by the standard $3+0$ scenario. The effort to explain the SBL anomalies (excess of electron-like events at low energy) has led to the models with possible presence of a sterile, fourth type of neutrino (this scenario is referred to as $3+1$ hereafter), which can have small mixing with the three active neutrinos. This fourth generation of neutrino, if exists, has to be sterile since the LEP experiment [31] restricts the number of active neutrino flavours to three. In addition to the six standard oscillation parameters mentioned above, $3+1$ scenario is parametrized by the mass squared difference $\Delta m_{41}^{2}$, three active-sterile mixing angles $\left(\theta_{14}, \theta_{24}, \theta_{34}\right)$, and two additional $\mathrm{CP}$ phases which we refer as $\delta_{24}$ and $\delta_{34}$. Recently the observation of a low energy electron-like event excess at a statistically significant $4.8 \sigma$ by the Mini Booster Neutrino Experiment (MiniBooNE) in its latest dataset [32, 33] has further boosted the search for sterile neutrinos. Various other existing and future facilities aim to search for sterile neutrinos with high precision using different neutrino sources and detection techniques. These facilities include IceCube [34], Karlsruhe Tritium Neutrino Experiment (KATRIN) [35], FermiLab's Short Baseline Neutrino (SBN) programme [36], ANTARES [37], Neutrino Experiment for Oscillation at Short baseline (NEOS) [38], Short baseline neutrino Oscillations with a novel Lithium-6 composite scintillator Detector(SoLid) [39], Neutrino-4 [40], Precision Reactor Oscillation and SPECTrum Experiment (PROSPECT) [41], Sterile Reactor Neutrino Oscillations (STEREO) [42, 43], Detector of the reactor AntiNeutrino based on Solid Scintillator (DANSS) [44], J-PARC Sterile Neutrino Search at J-PARC Spallation Neutron Source $\left(\mathrm{JSNS}^{2}\right)[45,46]$. Neutrino-4 has recently claimed to observe active-sterile oscillation at $3.5 \sigma$ around the vicinity of $\Delta m_{41}^{2} \approx 7 \mathrm{eV}^{2}$ and $\theta_{14} \approx 18^{\circ}$ by analyzing the its reactor antineutrino data accumulated since 2016 [47-49]. Though it has later been argued in literature that considering a more appropriate log-likelihood distribution [50, 51] or correct energy resolution [52], the statistical significance of the active-sterile observa- 
tion results in reactor neutrino experiments actually come down to a much lower value. ANTARES with its 10 years of data have found very mild $(\sim 1.6 \sigma)$ signature of sterile neutrino in their analyses [53]. Experiments looking for Coherent Elastic Neutrino-Nucleus Scattering (CE $\nu \mathrm{NS})$ [54] can also act as very useful probes of sterile neutrinos, as shown by the authors of [55].

The effect of sterile neutrino, should it exist with $\Delta m_{41}^{2} \sim 1 \mathrm{eV}^{2}$, is most pronounced around $L / E \sim 1 \mathrm{~km} / \mathrm{GeV}[56,57]$. In LBL experiments where $L / E \sim 500 \mathrm{~km} / \mathrm{GeV}$ at the far detectors (FD), the high frequency induced by $\Delta m_{41}^{2} \sim 1 \mathrm{eV}^{2}$ gets averaged out due to the finite energy resolution of the detector. Nevertheless, it has been shown $[56,58-80]$ that even at the FDs of these LBL experiments, the interference effects provided by the additional $\mathrm{CP}$ phases play very significant roles in spoiling the sensitivities to the crucial issues of $\mathrm{CPV}, \mathrm{MH}$ and $\theta_{23}$ octant. ${ }^{1}$ For instance, as shown in [64], the CPV sensitivity becomes a wide band whose width depends on the unknown magnitudes of the sterile phases $\delta_{24}$ and $\delta_{34},-$ leading to serious confusion in interpreting the results as $\mathrm{CP}$ violation or $\mathrm{CP}$ conservation. The constraints on the active-sterile mixing angles $\theta_{i 4}(i=1,2,3)$ do of course reduce such ambiguities in the interpretation of the results to some extent. But, a clear idea about how the LBL experiments are able to measure the sterile phases $\delta_{24}$ and $\delta_{34}$ given the constraints on the active-sterile mixing angles, will certainly minimise the obfuscation when their data are analyzed with a view to tackle the unresolved physics issues. The issue of measurement of one sterile phase ( $\delta_{24}$ or $\delta_{14}$, depending on the parametrization used) has been addressed to some extent in literature. [64] analyzes how the sterile phases impact the standard CPV and mass ordering measurements and also probe the joint parameter space $\delta_{13}-\delta_{24}$ for DUNE. The authors of [62] use a slightly different parameterization and discuss the CPV arising from the individual sterile $\mathrm{CP}$ phases and probe the joint parameter space $\delta_{13}-\delta_{14}$ at DUNE. They further extend their analysis by combining simulated data from other LBL experiments such as T2HK [72] and $\mathrm{ESS} \nu \mathrm{SB}$ [77]. Exploring the parameter space of $\delta_{13}-\delta_{24}$ has also been addressed in [71] for DUNE, T2HK, T2HKK and their combinations. The authors of [71] further illustrate how the individual phase $\delta_{24}$ can be measured by these experiments at various C.L. by assuming four possible true values $(0, \pm \pi / 2, \pi)$. [80] shows how the recent data from $\mathrm{T} 2 \mathrm{~K}$ and $\mathrm{NO} \nu \mathrm{A}$ can help to probe the parameter space of $\delta_{13}-\delta_{14}$. Most recently, the authors of [84] estimate how the difference $\left(\delta_{14}-\delta_{24}\right)$ in the sterile phases can impact in constraining the standard $\delta_{13}-\theta_{23}$ parameter space.

It is noteworthy that the sterile $\mathrm{CP}$ phase $\delta_{34}$ and its correlation with the other phases has been little addressed in literature. In the present manuscript we tackle the very relevant issue of estimating the capability to reconstruct all three CP phases $\left(\delta_{13}, \delta_{24}, \delta_{34}\right)$, taking into consideration their $\Delta \chi^{2}$ correlations with each other and also with the active-sterile mixing angles $\left(\theta_{14}, \theta_{24}, \theta_{34}\right)$. We carry out this exercise in the context of DUNE and illustrate the improvement when combined with $\mathrm{T} 2 \mathrm{~K}, \mathrm{NO} \nu \mathrm{A}$ (both these currently running experiments are simulated upto their present exposure) and T2HK. Apart from studying

\footnotetext{
${ }^{1}$ For a recent comprehensive status report of the impact of a light sterile neutrino in probing these issues at LBL see, for e.g., [81-83] and the references therein.
} 
these $\mathrm{CP}$ phases in detail, another crucial aspect in which our analysis differs from the existing studies mentioned above is that we have taken into consideration the current $3 \sigma$ hint of $\mathrm{CP}$ violation and the corresponding exclusion region of the CP phase $\delta_{13}$ by T2K data [14]. Moreover, in addition to illustrating how the 2-d parameter spaces for the CP phases can be probed, we also analyze how the individual CP phases can be reconstructed (after marginalizing all other relevant parameters) by the experiments, irrespective of the actual value they might have in nature. Additionally we have also considered the $\nu_{\mu} \rightarrow \nu_{\tau}$ channel (in addition to $\nu_{\mu} \rightarrow \nu_{e}$ and $\nu_{\mu} \rightarrow \nu_{\mu}$ ) in our study and estimated the capability of the projected data to measure all three $\mathrm{CP}$ phases in detail. This enables us to probe the parameter spaces associated to $\theta_{34}$ and $\delta_{34}$ with better sensitivity.

The present manuscript is organised as follows. In section 2 we give a brief account of the constraints on the sterile neutrino parameters and how the CP phases affect the relevant probabilities. In section 3 we describe the methodology of our statistical analyses. In section 4 we discuss the $\Delta \chi^{2}$ correlations among various CP phases, taking a pair of phases at a time. We also discuss the potential to reconstruct the three $\mathrm{CP}$ phases for all possible true values by performing simulations of DUNE, T2K, NOvA and T2HK. The role of individual oscillation channels in such reconstructions are analyzed in section 5 . We estimate the $\Delta \chi^{2}$ correlations among all the CP phases and the active-sterile mixing angles in section 6 . Finally in section 7 we briefly discuss how the relevant parameter spaces associated to Neutrinoless Double Beta Decay gets modified in light of the constraints on one eV scale sterile neutrino, followed by conclusion.

\section{Basics}

We first discuss the oscillation probabilities for the three channels $\left(P\left(\nu_{\mu} \rightarrow \nu_{e}\right), P\left(\nu_{\mu} \rightarrow \nu_{\mu}\right)\right.$ and $\left.P\left(\nu_{\mu} \rightarrow \nu_{\tau}\right)\right)$ in $3+1$ scenario. Since the expressions become immensely complicated in matter, we show them in vacuum and these will act as useful templates for gaining the physics insights in explaining our subsequent sensitivity results. For the mixing matrix we follow the parametrization scheme adopted in [56]:

$$
U^{3+1}=R\left(\theta_{34}, \delta_{34}\right) R\left(\theta_{24}, \delta_{24}\right) R\left(\theta_{14}\right) R\left(\theta_{13}\right) R\left(\theta_{23}, \delta_{13}\right) R\left(\theta_{12}\right)
$$

where $R\left(\theta_{i j}, \delta_{i j}\right)$ is a rotation in the $i j$-th plane with an associated phase $\delta_{i j}$ such that, for e.g.,

$$
R\left(\theta_{34}, \delta_{34}\right)=\left(\begin{array}{cccc}
1 & 0 & 0 & 0 \\
0 & 1 & 0 & 0 \\
0 & 0 & \cos \theta_{34} & e^{-i \delta_{34} \sin \theta_{34}} \\
0 & 0 & -e^{i \delta_{34} \sin \theta_{34}} & \cos \theta_{34}
\end{array}\right)
$$

Now we discuss briefly on the allowed ranges of the sterile sector parameters, as estimated in great detail in the global analysis of various neutrino data [85]. $\left|U_{e 4}\right|^{2}$ is bounded by $\nu_{e}$ and $\bar{\nu}_{e}$ disappearance searches and is equal to $\sin ^{2} \theta_{14}$. The combined atmospheric neutrino data from IceCube, DeepCore and SK, at 99\% C.L. (2 DOF) put the bound $\left|U_{e 4}\right|^{2} \lesssim 0.1$. This implies $\theta_{14} \lesssim 18.4^{\circ}$. It can be seen from all $\nu_{e}$ and $\bar{\nu}_{e}$ searches that the 
best fit value of $\left|U_{e 4}\right|^{2}$ is approximately equal to 0.01 , which gives $\theta_{14} \approx 5.7^{\circ}$. The data from $\nu_{\mu}$ and $\bar{\nu}_{\mu}$ disappearance searches put the following $99 \%$ C.L. (2 D.O.F.) constraints: $\left|U_{\mu 4}\right|^{2} \lesssim 0.01$ and $\left|U_{\tau 4}\right|^{2} \lesssim 0.17$. Since, in our parametrization $\left|U_{\mu 4}\right|=\cos \theta_{14} \sin \theta_{24}$ and $\left|U_{\tau 4}\right|=\cos \theta_{14} \cos \theta_{24} \sin \theta_{34}$, the corresponding bounds on $\theta_{24}$ and $\theta_{34}$ can easily be translated to $\theta_{24} \lesssim 6.05^{\circ}$ and $\theta_{34} \lesssim 25.8^{\circ}$. The allowed values of $\Delta m_{41}^{2}$ roughly lie in the range of $1-10 \mathrm{eV}^{2}$ and we consider it to be $1.3 \mathrm{eV}^{2}$ in the present work as per the global analysis. ${ }^{2}$

Using the standard approach for deriving oscillation probability, we obtain for the $\nu_{\alpha} \rightarrow \nu_{\beta}(\alpha, \beta=e, \mu, \tau, s$ and $\alpha \neq \beta)$ transition probability,

$$
\begin{aligned}
P_{\alpha \beta}^{3+1}= & 4\left|U_{\alpha 4} U_{\beta 4}\right|^{2} \times 0.5 \\
& -4 \operatorname{Re}\left(U_{\alpha 1} U_{\beta 1}^{*} U_{\alpha 2}^{*} U_{\beta 2}\right) \sin ^{2} \Delta_{21}+2 \operatorname{Im}\left(U_{\alpha 1} U_{\beta 1}^{*} U_{\alpha 2}^{*} U_{\beta 2}\right) \sin 2 \Delta_{21} \\
& -4 \operatorname{Re}\left(U_{\alpha 1} U_{\beta 1}^{*} U_{\alpha 3}^{*} U_{\beta 3}\right) \sin ^{2} \Delta_{31}+2 \operatorname{Im}\left(U_{\alpha 1} U_{\beta 1}^{*} U_{\alpha 3}^{*} U_{\beta 3}\right) \sin 2 \Delta_{31} \\
& -4 \operatorname{Re}\left(U_{\alpha 2} U_{\beta 2}^{*} U_{\alpha 3}^{*} U_{\beta 3}\right) \sin ^{2} \Delta_{32}+2 \operatorname{Im}\left(U_{\alpha 2} U_{\beta 2}^{*} U_{\alpha 3}^{*} U_{\beta 3}\right) \sin 2 \Delta_{32},
\end{aligned}
$$

where $\Delta_{i j}=\frac{\Delta m_{i j}^{2} L}{4 E}$. In deriving eq. (2.3), we have used the unitarity of the $4 \times 4$ mixing matrix $U$ and applied the usual assumptions that the term containing mass square splitting between $m_{4}$ and $m_{i}(i=1,2,3)$, i.e., $\sin ^{2} \Delta_{4 i}$ and $\sin 2 \Delta_{4 i}$ average out to 0.5 and 0 respectively at long baseline $(i=1,2,3)$. From eq. (2.3) one can use the long baseline approximation (i.e., neglecting the oscillation effects due to $\Delta m_{21}^{2}$ ) and arrive at the following simplified expression for the dominant channel $\nu_{\mu} \rightarrow \nu_{e}$.

$$
\begin{aligned}
P_{\mu e}^{4 \nu} \approx & \frac{1}{2} \sin ^{2} 2 \theta_{\mu e}^{4 \nu} \\
& +\left(a^{2} \sin ^{2} 2 \theta_{\mu e}^{3 \nu}-\frac{1}{4} \sin ^{2} 2 \theta_{13} \sin ^{2} 2 \theta_{\mu e}^{4 \nu}\right) \sin ^{2} \Delta_{31} \\
& +\cos \left(\delta_{13}+\delta_{24}\right) a \sin 2 \theta_{\mu e}^{3 \nu} \sin 2 \theta_{\mu e}^{4 \nu} \cos 2 \theta_{13} \sin ^{2} \Delta_{31} \\
& +\frac{1}{2} \sin \left(\delta_{13}+\delta_{24}\right) a \sin 2 \theta_{\mu e}^{3 \nu} \sin 2 \theta_{\mu e}^{4 \nu} \sin 2 \Delta_{31},
\end{aligned}
$$

where we have followed the convention of [56] for the following quantities.

$$
\begin{aligned}
\sin 2 \theta_{\mu e}^{3 \nu} & =\sin 2 \theta_{13} \sin \theta_{23}, \\
b & =\cos \theta_{13} \cos \theta_{23} \sin \theta_{12}, \\
\sin 2 \theta_{\mu e}^{4 \nu} & =\sin 2 \theta_{14} \sin \theta_{24}, \\
a & =\cos \theta_{14} \cos \theta_{24} .
\end{aligned}
$$

Eq. (2.4) tells us that in vacuum $P\left(\nu_{\mu} \rightarrow \nu_{e}\right)$ is sensitive to both $\delta_{13}$ and $\delta_{24}$, but not to $\delta_{34}$. As explained in [56], small dependence on $\delta_{34}$ creeps in when matter effect is taken into account. The expressions for the less dominant channels $P\left(\nu_{\mu} \rightarrow \nu_{\mu}\right)$ and $P\left(\nu_{\mu} \rightarrow \nu_{\tau}\right)$ can similarly be derived from eq. (2.3), but the expressions are quite lengthy. Interested readers can see $[75,86]$ for those probability expressions.

\footnotetext{
${ }^{2}$ In our notation, $m_{1,2,3}$ are the masses of the three active neutrinos, and $m_{4}$ denotes the mass of the sterile neutrino. Also, $\Delta m_{i j}^{2}=m_{i}^{2}-m_{j}^{2}$.
} 

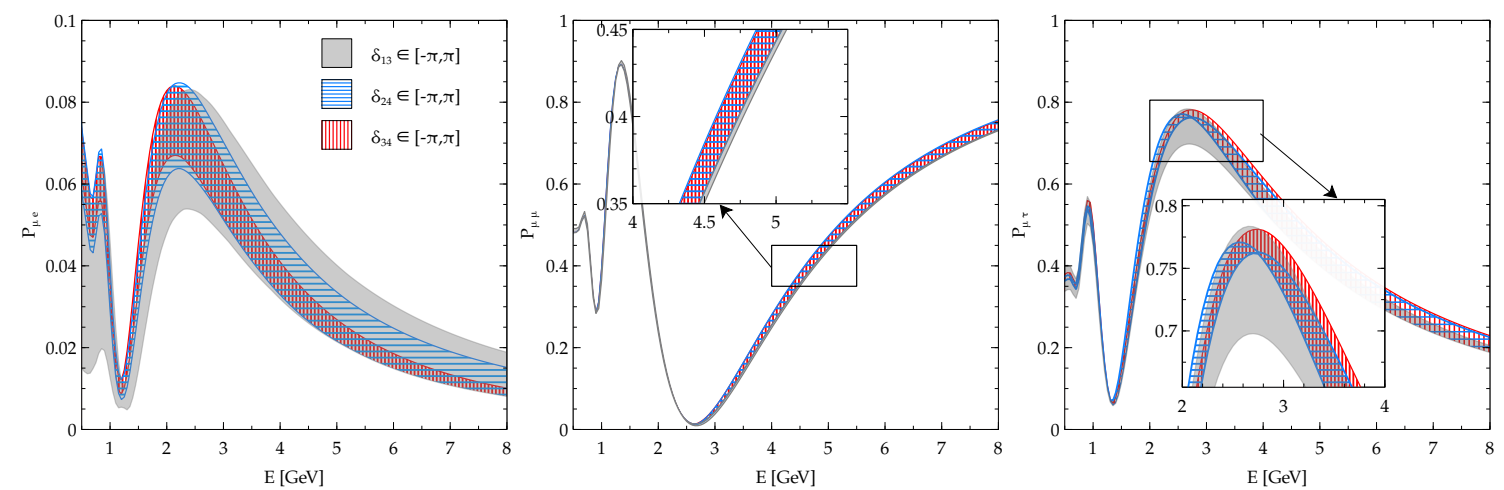

Figure 1. We show the probability bands due to individual variation of the CP phases $\delta_{13}$ (grey), $\delta_{24}$ (blue) and $\delta_{34}$ (red) in the whole range of $[-\pi, \pi]$ at a baseline of $1300 \mathrm{~km}$. The three panels correspond to the three channels $P\left(\nu_{\mu} \rightarrow \nu_{e}\right), P\left(\nu_{\mu} \rightarrow \nu_{\mu}\right)$ and $P\left(\nu_{\mu} \rightarrow \nu_{\tau}\right)$. The insets in the second and third panels show magnified versions of the rectangular regions indicated. The three active-sterile mixing angles were taken as $\theta_{14}=10^{\circ}, \theta_{24}=6^{\circ}, \theta_{34}=25^{\circ}$. The values of the rest of the oscillation parameters were taken from table 1 . Normal hierarchy was assumed for generating this plot.

Using the widely used General Long Baseline Experiment Simulator (GLoBES) [87, 88] and the relevant plugin snu.c $[89,90]$ for implementing sterile neutrinos, we now illustrate how the probabilities for different oscillation channels depends on the three $\mathrm{CP}$ phases $\left(\delta_{13}, \delta_{24}, \delta_{34}\right)$ individually at the DUNE baseline of $1300 \mathrm{~km}$. In figure 1 , we plot the bands for $P_{\mu e}, P_{\mu \mu}, P_{\mu \tau}$ (in the three panels respectively) due to individual variation of $\delta_{13}, \delta_{24}, \delta_{34}$, each being varied in the range $[-\pi, \pi]$. The grey band shows the variation of the standard Dirac CP phase $\delta_{13}$. The variation due to $\delta_{24}\left(\delta_{34}\right)$ is shown with the blue (red) band. For the variation of each CP phase, the other two phases are kept fixed: $\delta_{13}$ is kept fixed at the bf value of $-0.8 \pi$ while $\delta_{24}, \delta_{34}$ were considered to be zero. The sterile phases are associated with the active-sterile mixing angles. We have a slightly high active-sterile mixing: $\theta_{14}=10^{\circ}, \theta_{24}=6^{\circ}, \theta_{34}=25^{\circ}$. As expected, ${ }^{3}$ the $\mathrm{CP}$ phases have larger impact on appearance channels rather than the disappearance channels. $\nu_{\mu} \rightarrow \nu_{e}$ channel is most affected by the variation of $\delta_{13}$. Among the sterile CP phases, $\delta_{24}$ has a visibly greater impact on $P\left(\nu_{\mu} \rightarrow \nu_{e}\right)$ than that of $\delta_{34}$. It is interesting to note this feature especially in light of the fact that the value of the active-sterile mixing angle $\theta_{24}$ (taken as $6^{\circ}$ in figure 1$)$ is almost 5 times smaller than $\theta_{34}\left(25^{\circ}\right)$. We also observe that with increase in energy the effect of $\delta_{34}$ on $P\left(\nu_{\mu} \rightarrow \nu_{e}\right)$ further reduces. $P\left(\nu_{\mu} \rightarrow \nu_{\tau}\right)$ is less prone to variation of the $\mathrm{CP}$ phases. Still it shows slight variation for all three $\mathrm{CP}$ phases with $\delta_{24}$ and $\delta_{34}$ having almost similar effects but less than that of $\delta_{13} . P\left(\nu_{\mu} \rightarrow \nu_{\mu}\right)$ on the other hand, shows almost negligible variation with the $\mathrm{CP}$ phases. After a brief description of the simulation procedure we will estimate the capability of LBL experiments to reconstruct these CP phases.

\footnotetext{
${ }^{3}$ In eq. (2.3) for $\alpha=\beta$, the imaginary part in the r.h.s. vanishes, diminishing the effect of the CP phases.
} 


\begin{tabular}{|c|c|c|c|}
\hline Parameter & Best-fit-value & $3 \sigma$ interval & $1 \sigma$ uncertainty \\
\hline$\theta_{12}$ [Deg.] & 34.3 & $31.4-37.4$ & $2.9 \%$ \\
$\theta_{13}(\mathrm{NH})[\mathrm{Deg}]$. & 8.58 & $8.16-8.94$ & $1.5 \%$ \\
$\theta_{13}(\mathrm{IH})[\mathrm{Deg}]$. & 8.63 & $8.21-8.99$ & $1.5 \%$ \\
$\theta_{23}(\mathrm{NH})[\mathrm{Deg}]$. & 48.8 & $41.63-51.32$ & $3.5 \%$ \\
$\theta_{23}(\mathrm{IH})[\mathrm{Deg}]$. & 48.8 & $41.88-51.30$ & $3.5 \%$ \\
$\Delta m_{21}^{2}\left[\mathrm{eV}^{2}\right]$ & $7.5 \times 10^{-5}$ & {$[6.94-8.14] \times 10^{-5}$} & $2.7 \%$ \\
$\Delta m_{31}^{2}(\mathrm{NH})\left[\mathrm{eV}^{2}\right]$ & $+2.56 \times 10^{-3}$ & {$[2.46-2.65] \times 10^{-3}$} & $1.2 \%$ \\
$\Delta m_{31}^{2}(\mathrm{IH})\left[\mathrm{eV}{ }^{2}\right]$ & $-2.46 \times 10^{-3}$ & $-[2.37-2.55] \times 10^{-3}$ & $1.2 \%$ \\
$\delta_{13}(\mathrm{NH})[\mathrm{Rad}]$. & $-0.8 \pi$ & {$[-\pi, 0] \cup[0.8 \pi, \pi]$} & - \\
$\delta_{13}(\mathrm{IH})[\mathrm{Rad}]$. & $-0.46 \pi$ & {$[-0.86 \pi,-0.1 \pi]$} & - \\
$\theta_{14}[\mathrm{Deg}]$. & $5.7,10$ & $0-18.4$ & $\sigma\left(\sin ^{2} \theta_{14}\right)=5 \%$ \\
$\theta_{24}[\mathrm{Deg}]$. & 5,6 & $0-6.05$ & $\sigma\left(\sin ^{2} \theta_{24}\right)=5 \%$ \\
$\theta_{34}$ [Deg.] & 20,25 & $0-25.8$ & $\sigma\left(\sin ^{2} \theta_{34}\right)=5 \%$ \\
$\delta_{24}$ [Rad.] & $0,-0.5 \pi$ & {$[-\pi, \pi]$} & - \\
$\delta_{34}$ [Rad.] & $0,-0.5 \pi$ & {$[-\pi, \pi]$} & - \\
\hline
\end{tabular}

Table 1. Standard oscillation parameters and their uncertainties used in our study. The values of $3+0$ parameters were taken from the global fit analysis in [22] while the $3+1$ parameter values were chosen from [85] (see also section 2). If the $3 \sigma$ upper and lower limit of a parameter is $x_{u}$ and $x_{l}$ respectively, the $1 \sigma$ uncertainty is $\left(x_{u}-x_{l}\right) / 3\left(x_{u}+x_{l}\right) \%$ [17]. For the active-sterile mixing angles, a conservative $5 \%$ uncertainty was used on $\sin ^{2} \theta_{i 4}(\mathrm{i}=1,2,3)$.

\section{Simulation details}

We simulate the long baseline neutrino experiments DUNE, NOvA, T2K and T2HK using GLoBES [87, 88]. DUNE is a $1300 \mathrm{~km}$ long baseline experiment employing a liquid argon far detector (FD) of $40 \mathrm{kt}$ fiducial mass with a beam of power 1.07 MW and running 3.5 years each on $\nu$ and $\bar{\nu}$ mode (resulting in a total exposure of roughly $300 \mathrm{kt}$.MW.yr corresponding to $1.47 \times 10^{21}$ protons on target or POT). We have used the official configuration files [91] provided by the DUNE collaboration for its simulation. Following this, we have also taken into account the presence of a near detector (ND) at $459 \mathrm{~m}$ from the source. The ND helps in making a more precise measurement of the flux and cross-section, thereby reducing the relevant systematic uncertainties at the FD. We should mention here that we have done a $\Delta \chi^{2}$ analysis (discussed later) with the simulated data at FD alone, rather than a joint $\Delta \chi^{2}$ analysis using simulated data both at ND and FD. ${ }^{4}$ Electron neutrino appearance signals (CC), muon neutrino disappearance signals (CC), as well as neutral current (NC) backgrounds and tau neutrino appearance backgrounds (along with the corresponding systematics/efficiencies etc.) are already included in the configuration files.

\footnotetext{
${ }^{4}$ It is worthwhile to note here that an $\mathrm{eV}$-scale sterile neutrino will have its signature at the ND due to short baseline active-sterile oscillation and consequently a joint analysis using both ND and FD data would probably constrain the active-sterile mixing angles slightly more. But our main aim is the analyses of the $\mathrm{CP}$ phases, given the already existing constraints on the mixing angles from the global analysis [85], and this would have more observable signals at the FD, especially in neutrino appearance measurements [56].
} 
In the present analysis, we have additionally incorporated tau neutrino appearance as a separate signal following $[75,76]$. Charged current interaction of an incoming $\nu_{\tau}$ produces a $\tau$ lepton (requires a threshold energy of $\gtrsim 3.4 \mathrm{GeV}$ for the incoming $\nu_{\tau}$ ), which can decay hadronically (with a branching fraction $\sim 65 \%$ ) or leptonically (with a branching fraction of $\sim 35 \%$ ). The analysis of the hadronic decay channel involves the capability of the detector to study the resulting pions and kaons. More importantly, NC neutrino scattering constitutes the biggest background for the hadronic decay channel of $\tau$. Following [75], we have used an efficiency to separate $30 \%$ hadronically decaying $\tau$ events (with about $1 \% \mathrm{NC}$ events remaining). On the other hand, the leptonic decay channels of $\tau\left(\tau^{-} \rightarrow e^{-} \bar{\nu}_{e} \nu_{\tau}\right.$; $\left.\tau^{-} \rightarrow \mu^{-} \bar{\nu}_{\mu} \nu_{\tau}\right)$ are more difficult to analyse, due to the large background mainly consisting of $\nu_{e}$-CC and $\nu_{\mu}$-CC respectively (along with backgrounds from NC and contaminations due to wrong sign leptons.). Following [76], we have taken the efficiency of the electron channel to be $15 \%$. Due to the overwhelming background, we have taken a nominal efficiency of $5 \%$ in the muon channel. Naturally, the contribution of the leptonic decay channel of $\tau$ is very small. We should also mention here that the decay of $\tau$ at the detector will involve missing energy in the form of an outgoing $\nu_{\tau}$, which in turn makes the energy reconstruction of the incoming $\nu_{\tau}$ difficult. From [75], we use a Gaussian energy reconstruction with a resolution of $20 \%$ which is a conservative estimate. We acknowledge that our implementation of the $\nu_{\tau}$ channel as a signal is conservative in nature. Nevertheless, this provides a small but non-negligible statistics in terms of events and $\Delta \chi^{2}$ sensitivity (see section 5 and appendix C). Using a much more sophisticated analysis of $\nu_{\tau}$ appearance channel at DUNE by implementing jet- clustering algorithms and machine learning techniques, as has been pioneered in [92], one certainly expects to exploit the rich physics capabilities hidden within this channel.

We have simulated NOvA with a baseline of $800 \mathrm{~km}$ employing an FD of fiducial mass of $14 \mathrm{kt}$ and a beam of $742 \mathrm{~kW}$. The simulation for NOvA was implemented according to [15] which generates $8.85 \times 10^{20}\left(12.33 \times 10^{20}\right)$ POT in $\nu(\bar{\nu})$ mode. T2K is a $295 \mathrm{~km}$ experiment with a $22.5 \mathrm{kt}$ water cherencov FD. For T2K simulation we use the inputs from $[14,93]$. We have used a beam of $515 \mathrm{~kW}$ and simulating $1.97 \times 10^{21}\left(1.63 \times 10^{21}\right)$ POT in $\nu(\bar{\nu})$ mode. T2HK is an upgraded version of T2K with a higher beam of 1.3 MW and a much bigger fiducial mass of $187 \mathrm{kt}$ of its water cherencov FD. For T2HK we simulate a total of $2.7 \times 10^{22}$ POT in 1:3 ratio of $\nu$ and $\bar{\nu}$ mode (with inputs taken from $[19,94]$ ). Note that, for the future experiments DUNE and T2HK we have used the full expected exposure, while for the currently running experiments $\mathrm{T} 2 \mathrm{~K}$ and NOvA we have simulated upto their current exposure.

To estimate the capability of LBL experiments to reconstruct the CP phases, we carry out a $\Delta \chi^{2}$ analysis using GLoBES and the relevant plugin snu.c [89, 90] for implementing sterile neutrinos. In order to gain insight, let us examine the analytical form of the $\Delta \chi^{2}$,

$$
\begin{aligned}
\Delta \chi^{2}\left(p^{\text {true }}\right)=\operatorname{Min}_{p^{\text {test }}, \eta} & {\left[2 \sum _ { k } ^ { \text { mode channel bin } } \sum _ { j } \sum _ { i } \left\{N_{i j k}^{\text {test }}\left(p^{\text {test }} ; \eta\right)-N_{i j k}^{\text {true }}\left(p^{\text {true }}\right)\right.\right.} \\
& \left.\left.+N_{i j k}^{\text {true }}\left(p^{\text {true }}\right) \ln \frac{N_{i j k}^{\text {true }}\left(p^{\text {true }}\right)}{N_{i j k}^{\text {test }}\left(p^{\text {test }} ; \eta\right)}\right\}+\sum_{l} \frac{\left(p_{l}^{\text {true }}-p_{l}^{\text {test }}\right)^{2}}{\sigma_{p_{l}}^{2}}+\sum_{m} \frac{\eta_{m}^{2}}{\sigma_{\eta_{m}}^{2}}\right],
\end{aligned}
$$


where $N^{\text {true }}\left(N^{\text {test }}\right)$ is the true (test) set of events, while $p^{\text {true }}\left(p^{\text {test }}\right)$ is the set of true (test) oscillation parameters. The index $i$ is summed over the energy bins of the experiment concerned (as discussed above). The indices $j$ and $k$ are summed over the oscillation channels $\left(\nu_{\mu} \rightarrow \nu_{e}, \nu_{\mu} \rightarrow \nu_{\mu}, \nu_{\mu} \rightarrow \nu_{\tau}\right)$ and the modes $(\nu$ and $\bar{\nu})$ respectively. The term $\left(N^{\text {test }}-N^{\text {true }}\right)$ takes into account the algebraic difference while the log-term inside the curly braces considers the fractional difference between the test and true sets of events. The term summed over $i, j, k$ and written inside the curly braces is the statistical part of $\Delta \chi^{2} . \sigma_{p_{l}}$ is the uncertainty in the prior measurement of the $l$-th oscillation parameter $p_{l}$. The values of the true or best-fit oscillation parameters and their uncertainties as used in the present analysis are tabulated in table 1 . In the last term, $\sigma_{\eta_{m}}$ is the uncertainty on the systematic/nuisance parameter $\eta_{m}$ and the sum over $m$ takes care of the systematic part of $\Delta \chi^{2}$. This way of treating the systematics in the $\Delta \chi^{2}$ calculation is known as the method of pulls [95-98]. For DUNE, the $\nu_{e}$ and $\bar{\nu}_{e}$ signal modes have a normalization uncertainties of $2 \%$ each, whereas the $\nu_{\mu}$ and $\bar{\nu}_{\mu}$ signals have a normalization uncertainty of $5 \%$ each. The $\nu_{\tau}$ and $\bar{\nu}_{\tau}$ signals have a normalization uncertainties of $20 \%$ each. The background normalization uncertainties vary from $5 \%-20 \%$ and include correlations among various sources of background (coming from beam $\nu_{e} / \bar{\nu}_{e}$ contamination, flavour misidentification, neutral current and $\nu_{\tau}$ ). The final estimate of $\Delta \chi^{2}$ obtained after a marginalization over the $3 \sigma$ range of test parameters ${ }^{5} p^{\text {test }}$ and the set of systematics $(\eta)$ is a function of the true values of the oscillation parameters. Technically this $\Delta \chi^{2}$ is the frequentist method of hypotheses testing [96, 99].

\section{Reconstruction of the CP phase in correlation with other phases}

In figure 2, we illustrate how the CP phases can be reconstructed at $1 \sigma$ C.L. in the plane of (test $\delta_{13}$-test $\left.\delta_{24}\right)$, (test $\delta_{13}$-test $\delta_{34}$ ) and (test $\delta_{24}$-test $\delta_{34}$ ) in the three columns respectively. The red contour shows the reconstruction capability of DUNE. The green and blue contours illustrate the reconstruction when it is combined with $(\mathrm{T} 2 \mathrm{~K}+\mathrm{NO} \nu \mathrm{A})$ and $(\mathrm{T} 2 \mathrm{~K}+\mathrm{NO} \nu \mathrm{A}$ $+\mathrm{T} 2 \mathrm{HK}$ ) respectively. The top (bottom) row of figure 2 shows the choice of the $\mathrm{CP}$ conserving (maximally $\mathrm{CP}$ violating) true values of $\delta_{24}$ and $\delta_{34}$. Marginalisation has been carried over the test values of $\theta_{13}, \theta_{23}, \Delta m_{31}^{2}$ (including both the mass hierarchies) with prior uncertainties mentioned in table 1 . the active-sterile mixing angles $\theta_{14}, \theta_{24}, \theta_{34}$ (with a prior uncertainty of $5 \%$ on each $\sin ^{2} \theta_{i 4}$ ) and the third CP phase not shown along the axes of a particular panel has also been considered for marginalisation. Clearly the reconstruction of $\delta_{13}$ is better (as evidenced by the narrowness of the contours along the test $\delta_{13}$ axis) compared to the other two phases. This happens since the associated mixing angle $\theta_{13}$ has been measured very precisely unlike the corresponding active-sterile mixing angles $\theta_{24}$ and $\theta_{34}$ (see table 1 and section 2). For the maximally CP violating choices of true $\delta_{24}$ and true $\delta_{34}$, their reconstruction gets better at the cost of small degeneracies appearing for DUNE around test $\delta_{24} \approx 135^{\circ}, \delta_{34} \approx 90^{\circ}$. Adding data from other experiments lifts these

\footnotetext{
${ }^{5}$ The test parameters that are marginalised include those listed in table 1 along with the specified ranges and priors. Note that, we have used a $5 \%$ prior for the sine of each of the active-sterile mixing angles while varying them over their entire range.
} 


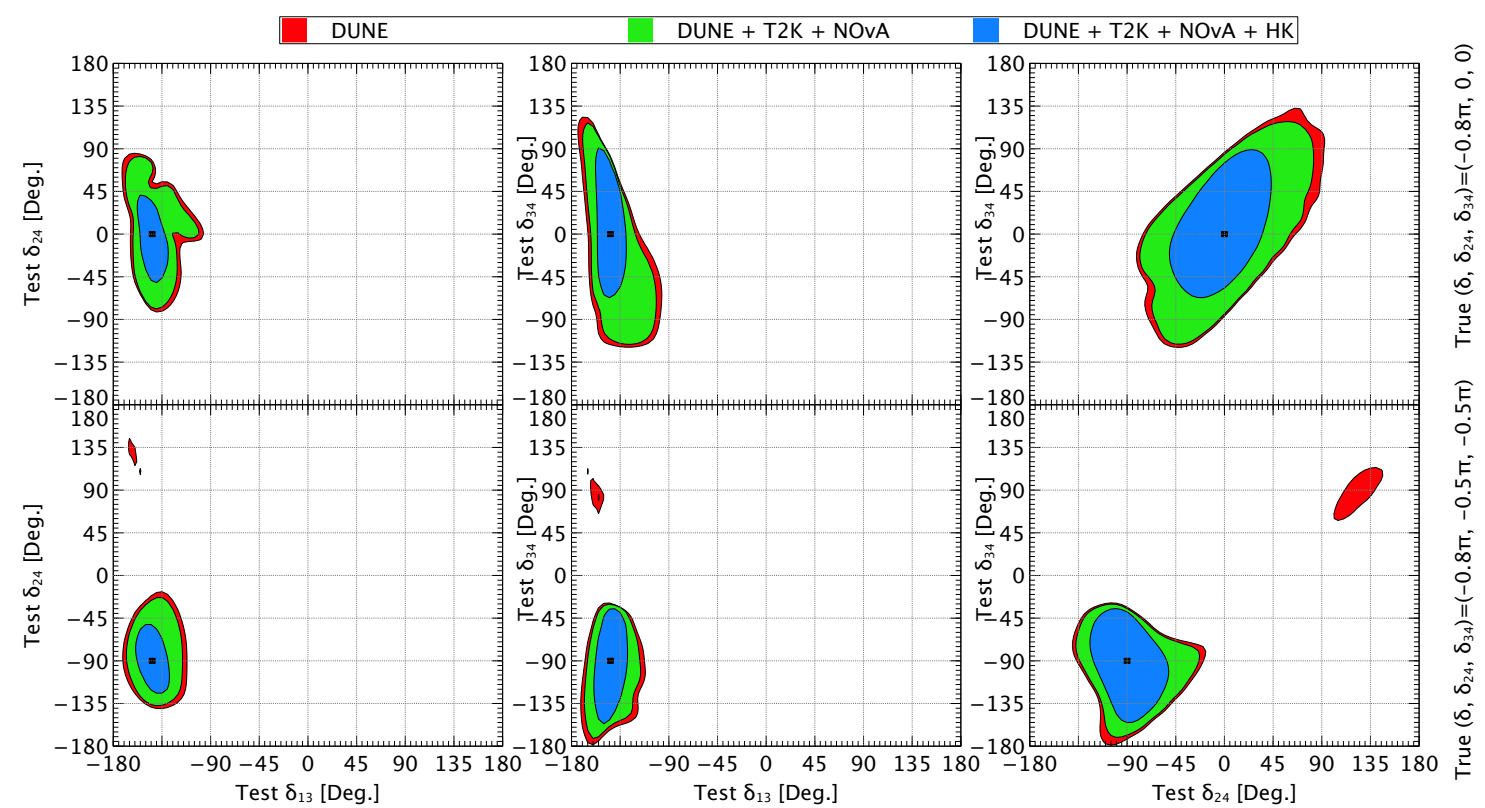

Figure 2. Reconstruction of the CP phases, taken pairwise at a time, at a C.L. of $1 \sigma$ (2 D.O.F.) at DUNE (red), DUNE + T2K + NO $\nu$ A (green), and DUNE + T2K + NO $\nu \mathrm{A}+\mathrm{T} 2 \mathrm{HK}$ (blue). The top (bottom) row corresponds to the choice $\delta_{24}=\delta_{34}=0(-\pi / 2)$. The true value of the standard Dirac CP phase $\delta_{13}$ is fixed at $-0.8 \pi$. The black dot indicates the true values assumed. The true values of active-sterile mixing angles are taken as $\theta_{14}, \theta_{24}, \theta_{34}=5.7^{\circ}, 5^{\circ}, 20^{\circ}$. Other oscillation parameters were taken from table 1.

degeneracies. We would also like to refer the reader to appendices $\mathrm{A}$ and $\mathrm{B}$, which show the effect of prior of $\theta_{23}$ and different true choices of the standard oscillation parameters on the reconstruction of the $\mathrm{CP}$ phases.

In figure 3, we illustrate how efficiently the combination of LBL experiments can reconstruct the three CP phases $\delta_{13}, \delta_{24}$ and $\delta_{34}$ at $1 \sigma$ C.L. in the three columns respectively given their true value lying anywhere in the whole parameter space of $[-\pi, \pi]$. In addition to the poorly measured $3+0$ parameters $\left(\theta_{23}, \Delta m_{31}^{2}\right)$ and the active-sterile mixing angles $\left(\theta_{14}, \theta_{24}, \theta_{34}\right)$, in each panel we have also marginalised over the two other CP phases $(\in[-\pi, \pi])$ not shown along the axes. The top (bottom) row depicts small (large) activesterile mixing with true $\theta_{14}, \theta_{24}, \theta_{34}=5.7^{\circ}, 5^{\circ}, 20^{\circ}\left(\theta_{14}, \theta_{24}, \theta_{34}=10^{\circ}, 6^{\circ}, 25^{\circ}\right)$. Note that, the latter choice of values represents the upper limits of the allowed active-sterile mixing (See section 2). For each true value of the CP phases $(\in[-\pi, \pi])$, the corresponding vertical width of the contours provide an estimate of the precision of reconstructing that true value. It is evident that in comparison to the reconstruction of the sterile phases, the standard Dirac phase $\delta_{13}$ can be reconstructed much more efficiently by the LBL experiments. We note that the reconstruction of $\delta_{13}$ does not noticeably depend upon the size of the activesterile mixing as assumed in the two rows. As the T2K data [14] suggests, if $\delta_{13}$ indeed turns out to lie in the lower half plane of $[-\pi, 0]$ with a best fit roughly around the maximal $\mathrm{CPV}(\approx-\pi / 2)$, the future analyses with a combination of (DUNE $+\mathrm{T} 2 \mathrm{~K}+\mathrm{NO} \nu \mathrm{A}+$ 

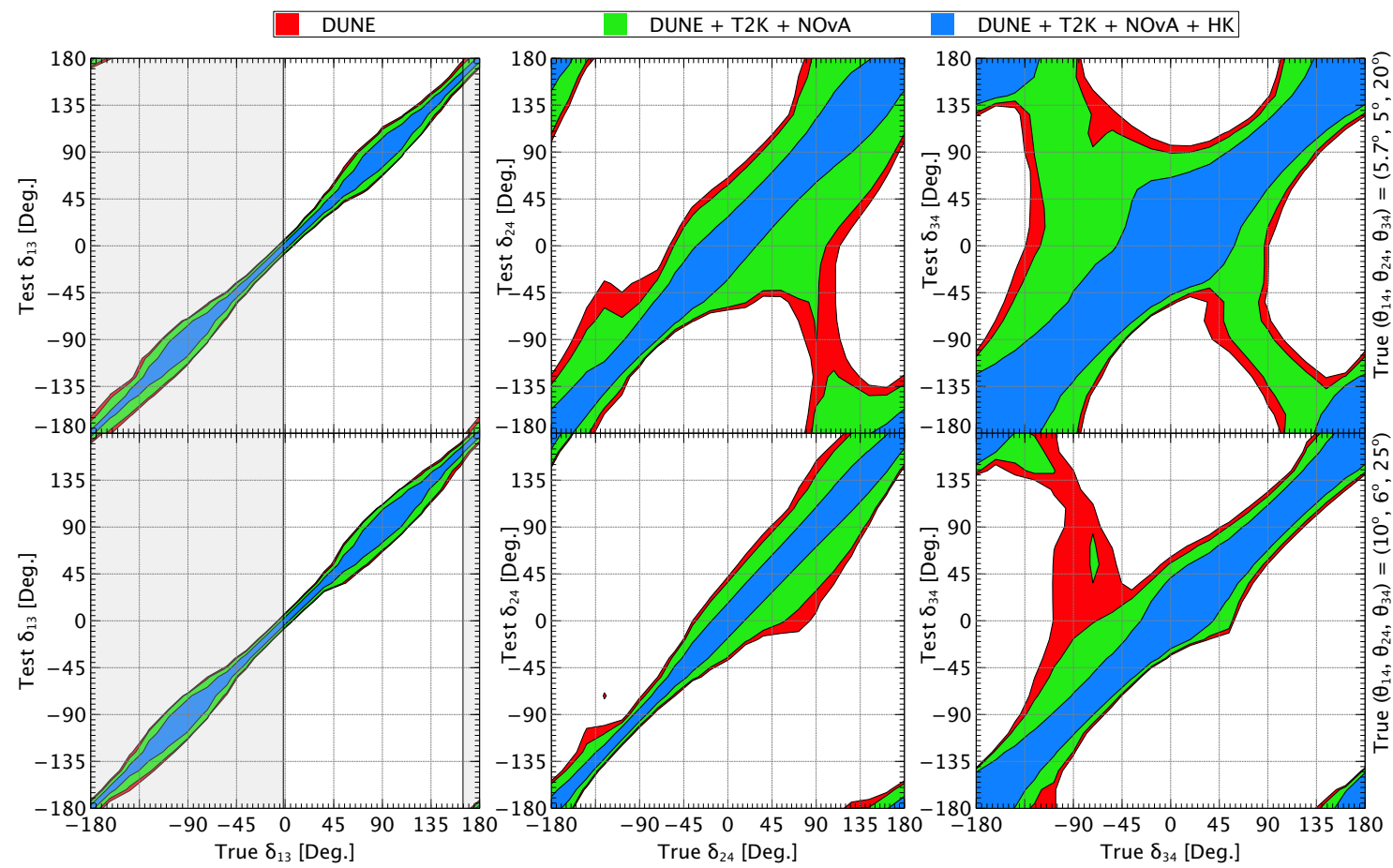

Figure 3. Reconstruction of the CP phases $\delta_{13}, \delta_{24}$ and $\delta_{34}$, for all the choices of their true values in $[-\pi, \pi]$ at a C.L. of $1 \sigma(1$ D.O.F.) at DUNE (red), DUNE + T2K + NO $\nu$ A (green), and DUNE + $\mathrm{T} 2 \mathrm{~K}+\mathrm{NO} \nu \mathrm{A}+\mathrm{T} 2 \mathrm{HK}$ (blue). The top (bottom) row corresponds to the choice of the active-sterile mixing angles as true $\theta_{14}, \theta_{24}, \theta_{34}=5.7^{\circ}, 5^{\circ}, 20^{\circ}\left(10^{\circ}, 6^{\circ}, 25^{\circ}\right)$. The true values of the phases not shown in a panel are fixed at: $\delta_{13}, \delta_{24}$ and $\delta_{34}=-0.8 \pi, 0$ and 0 respectively. In the first column the grey shaded regions depict the $3 \sigma$ allowed values measured by T2K [14].

T2HK) with their projected runtime will be able to measure this phase in a narrow approximate range of $\left[-115^{\circ},-75^{\circ}\right]$ (at $\left.1 \sigma\right)$. The precision is marginally better if $\delta_{13}$ turns out to be close to the $\mathrm{CP}$ conserving value (0). As far as the reconstruction of the sterile phases are concerned, $\delta_{24}$ can be reconstructed with a better precision than $\delta_{34}$. For small activesterile mixing (top row of figure 3 ) and without considering the T2HK-projected data, $\delta_{24}$ can be better reconstructed only in the lower half plane. But with T2HK-projected data, its reconstruction becomes much better throughout the entire parameter space. T2HK, due to its shorter baseline and much higher fiducial mass of its water cerenkov detector can offer very high statistics which helps to alleviate the degeneracy. On the other hand, the reconstruction of $\delta_{34}$ is bad almost for the entire parameter space if T2HK is not considered. For e.g., if the true value of $\delta_{34}$ in nature turns out to be around $\left[-135^{\circ},-90^{\circ}\right.$, the range of values reconstructed at $1 \sigma$ by the combination of DUNE $+\mathrm{T} 2 \mathrm{~K}+\mathrm{NO} \nu \mathrm{A}$ can be anywhere between $-180^{\circ}$ to $180^{\circ}$. Large active-sterile mixing (bottom row of figure 3 ) significantly improves the sensitivities of the LBL experiments to the sterile CP phases, resulting in significant improvement of the reconstruction of $\delta_{24}$ and $\delta_{34}$. We have already seen in figure 1 that $P\left(\nu_{\mu} \rightarrow \nu_{e}\right)$ is most sensitive to $\delta_{13}$ and least sensitive to $\delta_{34}$. This leads to a better precision in reconstructing $\delta_{13}$ in comparison to the others. 

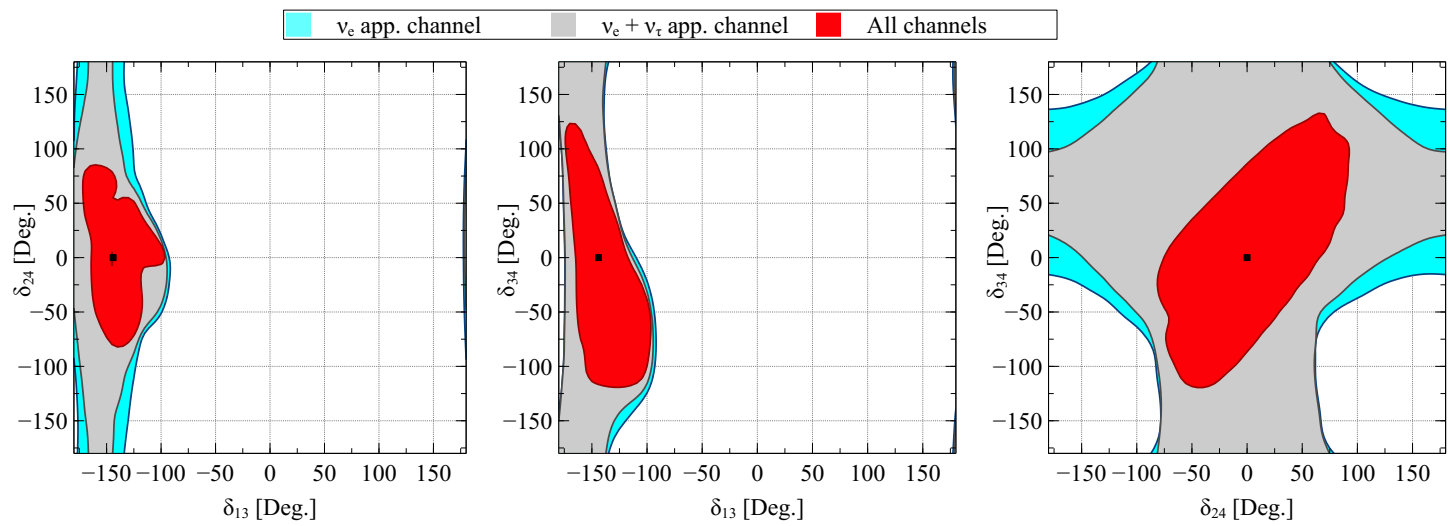

Figure 4. Reconstruction of the $\mathrm{CP}$ phases, taken pairwise at a time for three different channels at DUNE at a C.L. of $1 \sigma$ (2 D.O.F.). for $\nu_{e}$ appearance channel (cyan), $\nu_{e}+\nu_{\tau}$ appearance channel (grey) and all channels (red). The three panels depict the test values of the three $\mathrm{CP}$ phases. The true values for the $\mathrm{CP}$ phases were assumed as $\delta_{13}, \delta_{24}, \delta_{34}=-0.8 \pi, 0,0$ and the true activesterile mixing angles were chosen as $\theta_{14}, \theta_{24}, \theta_{34}=5.7^{\circ}, 5^{\circ}, 20^{\circ}$. The black dot indicates the true values assumed.

\section{Role of individual channels in reconstruction}

Figure 4 illustrates the impact of different appearance and disappearance channels on the reconstruction of the $\mathrm{CP}$ phases (taken pairwise, like in figure 2) at $1 \sigma$ C.L. at DUNE. The innermost red contours consisting of all the three channels for DUNE are the same as the red contours in the upper row of figure 2 . We can clearly see the decrease in uncertainty in the measurement of the $\mathrm{CP}$ phases as we keep on adding $\nu_{\mu} \rightarrow \nu_{\tau}$ appearance and $\nu_{\mu} \rightarrow \nu_{\mu}$ disappearance channel to the $\nu_{\mu} \rightarrow \nu_{e}$ appearance channel. The phase dependence of the $\nu_{\mu} \rightarrow \nu_{\tau}$ channel (see also figure 1) helps somewhat in this case. Note that, the improvement in result due to the addition of the $\nu_{\tau}$ appearance channel is not very significant and this is due to the very low statistics provided by this channel due to the difficulty in observing $\nu_{\tau}$. On the other hand, though the phase dependence of $\nu_{\mu} \rightarrow \nu_{\mu}$ channel is small, it offers a very large number of events compared to the other two channels, thereby decreasing the uncertainty in reconstruction (see also, appendix C). The improvement in reconstruction along the $\delta_{13}$ direction is not significant with the addition of channels, underlining the pivotal role here played by the $\nu_{e}$ appearance channel. In contrast, as observed from the first two panels of figure 4 , the reconstruction of $\delta_{24}$ and $\delta_{34}$ is significantly better (more so in the former) particularly by the addition of $\nu_{\mu}$ disappearance channel. In the third panel, the role of $\nu_{\mu}$ disappearance is emphasized by the shrinking of the reconstruction contour in both $\delta_{24}$ and $\delta_{34}$ directions. Here we also note a slight improvement in reconstruction along the $\delta_{34}$ axis (but not so much along $\delta_{24}$ ) with the addition of $\nu_{\tau}$ appearance channel. In the next section we are going to estimate the correlations of the phases with the active-sterile mixing angles. 


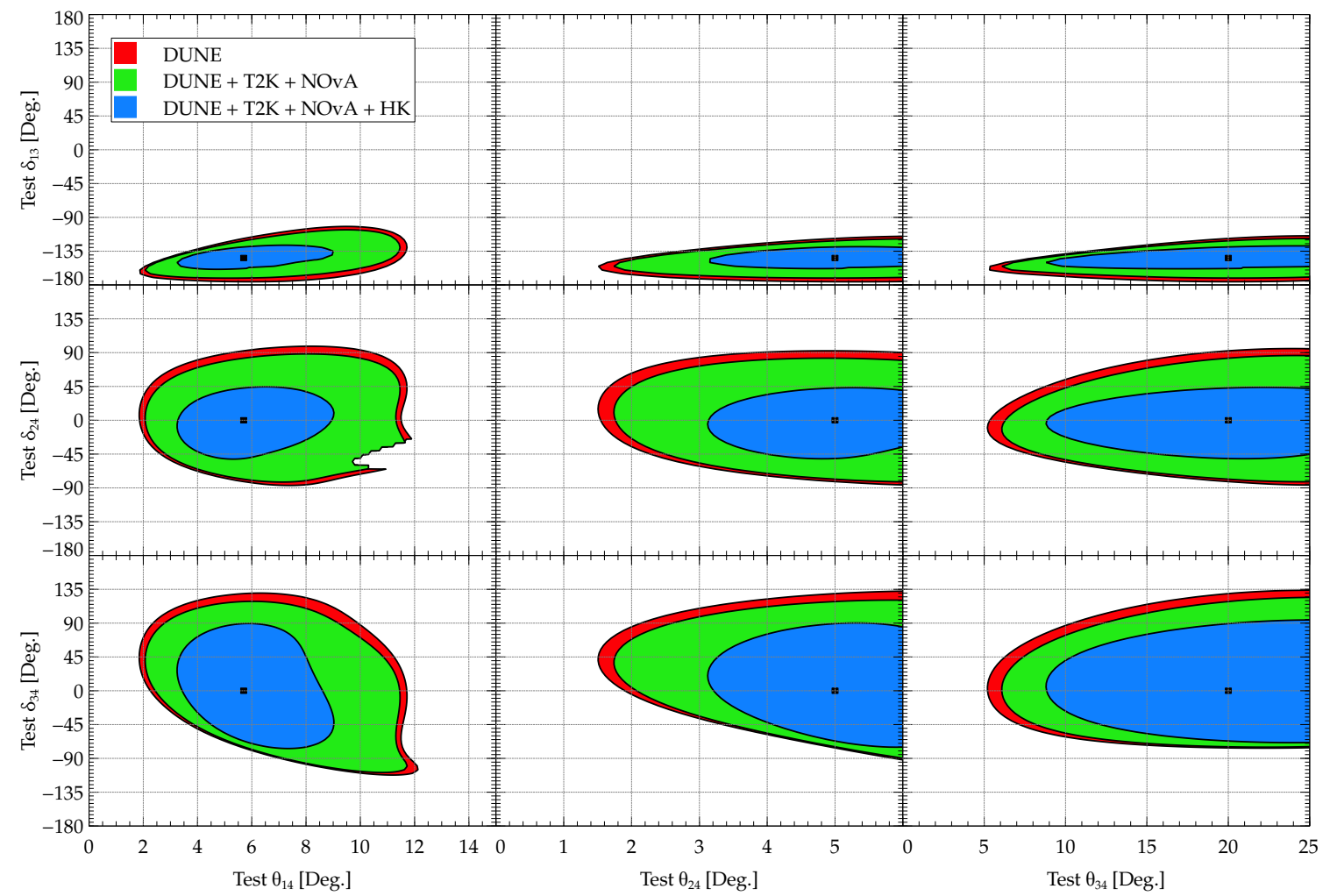

Figure 5. Reconstruction of the $\mathrm{CP}$ phases in correlation with the mixing angles $\theta_{14}, \theta_{24}, \theta_{34}$ at a C.L. of $1 \sigma$ (2 D.O.F.) at DUNE (red), DUNE + T2K + NO $\nu$ A (green), and DUNE + T2K + $\mathrm{NO} \nu \mathrm{A}+\mathrm{T} 2 \mathrm{HK}$ (blue). The three columns (rows) depict the test values of the three active-sterile mixing angles (three CP phases). The true values for the $\mathrm{CP}$ phases were assumed as $\delta_{13}, \delta_{24}, \delta_{34}=$ $-0.8 \pi, 0,0$ and the true active-sterile mixing angles were chosen as $\theta_{14}, \theta_{24}, \theta_{34}=5.7^{\circ}, 5^{\circ}, 20^{\circ}$. The black dot indicates the true values assumed.

\section{Reconstruction of the CP phase in correlation with active-sterile mixing angles}

In figure 5 we show how efficiently the LBL experiments can reconstruct the CP phases in correlation with active-sterile mixing angles at $1 \sigma$ C.L. (2 D.O.F.). The three phases (angles) are shown along the three rows (columns). In the second and third column the ranges of $\theta_{24}$ and $\theta_{34}$ are not shown beyond their allowed values. This results in the contours not being closed in these cases. As expected, the top row shows much less uncertainty along the test $\delta_{13}$ direction for all the three angles involved. In all the panels, we note that combining $\mathrm{NO} \nu \mathrm{A}$ and $\mathrm{T} 2 \mathrm{~K}$ data to DUNE gives mild improvement while a further addition of data projected by T2HK significantly improves the reconstruction. We have varied the test values of the angles upto their upper limit at $99 \%$ C.L.: $\theta_{14} \lesssim 18.4^{\circ}, \theta_{24} \lesssim 6.05^{\circ}, \theta_{34} \lesssim 25.8^{\circ}$, and the true values to be reconstructed were $\theta_{14}=5.7^{\circ}, \theta_{24}=5^{\circ}, \theta_{34}=20^{\circ}$ (as discussed in section 2).

The horizontal (vertical) span of the resulting contours indicate the uncertainties along the mixing angles (CP phases). We observe that the contours involving $\theta_{24}$ and $\theta_{34}$ do not 


\begin{tabular}{|c|c|c|}
\hline Angle & $\begin{array}{c}\text { Value to be reconstructed } \\
\text { [Degree] }\end{array}$ & $\begin{array}{c}\text { Reconstructed range } \\
\text { [Degree] }\end{array}$ \\
\hline$\theta_{14}$ & 5.7 & $3.3 \lesssim \theta_{14} \lesssim 9.1$ \\
\hline$\theta_{24}$ & 5 & $3.2 \lesssim \theta_{24} \lesssim 6$ \\
\hline$\theta_{34}$ & 20 & $9.0 \lesssim \theta_{34} \lesssim 25$ \\
\hline
\end{tabular}

Table 2. The maximum reconstructed ranges for $\theta_{14}, \theta_{24}, \theta_{34}$ as estimated from figure 5 for DUNE $+\mathrm{T} 2 \mathrm{~K}+\mathrm{NOvA}+\mathrm{HK}$.

close as long as we confine ourselves upto the allowed upper limit of $\theta_{24}$ and $\theta_{34}$. It is interesting to note that even if $\theta_{34}$ is allowed to vary in a larger range, the contours involving it show considerable uncertainty. This signifies the difficulty in constraining $\theta_{34}$ in the experiments. It is mainly this reason which also hinders a good reconstruction of the associated phase $\delta_{34}$, as we have seen in section 4 . To have a quantitative idea about the potential to reconstruct the true values of the active-sterile mixing angle $\theta_{i 4}(i=1,2,3)$, we calculate how much the total horizontal span of each blue contour is. From this we estimate the maximum range of uncertainty (so as to obtain a conservative range) in reconstructing $\theta_{i 4}$ and tabulate these below in table 2 .

\section{Impact of constraints on neutrinoless double beta decay}

Before concluding, we include discussion of $3+1$ framework on other observables, such as, neutrinoless double beta decay. The sterile neutrino, if a Majorana particle, gives non-zero contribution in the lepton number violating neutrinoless double beta decay (NDBD). In the presence of a non-zero $\theta_{14}$, the effective mass of NDBD process becomes [100],

$$
m_{\mathrm{eff}}=\left.\left|m_{1}\right| U_{e 1}\right|^{2}+m_{2}\left|U_{e 2}\right|^{2} e^{i \alpha_{2}}+m_{3}\left|U_{e 3}\right|^{2} e^{i \alpha_{3}}+m_{4}\left|U_{e 4}\right|^{2} e^{i \alpha_{4}} \mid,
$$

where $\alpha_{2}, \alpha_{3}, \alpha_{4}$ are the relevant CP phases. Following the parametrisation given in eq. (2.1), the relevant elements of the mixing matrix are as follows.

$$
\left|U_{e 1}\right|=c_{12} c_{13} c_{14},\left|U_{e 2}\right|=s_{12} c_{13} c_{14},\left|U_{e 3}\right|=s_{13} c_{14},\left|U_{e 4}\right|=\sin \theta_{14} .
$$

The expression for half-life of $0 \nu \beta \beta$ transition can be given as [101],

$$
\frac{1}{T_{1 / 2}^{0 \nu}}=G_{0 \nu}\left|M_{\nu} \eta_{\nu}+M_{N} \eta_{N}\right|^{2}
$$

where,

$$
\eta_{\nu}=\frac{U_{e i}^{2} m_{i}}{m_{e}}, \quad \eta_{N}=\frac{V_{e i}^{2} m_{p}}{M_{i}}
$$

In the above, $m_{i}$ is the mass of active neutrino and $U_{e i}$ is the PMNS mixing; whereas, $\theta_{e i}$ is the mixing among the active and sterile and $M_{i}$ is the corresponding mass of heavy sterile. In the above, $M_{\nu}$ and $M_{N}$ are the nuclear matrix elements (NME) for exchange 

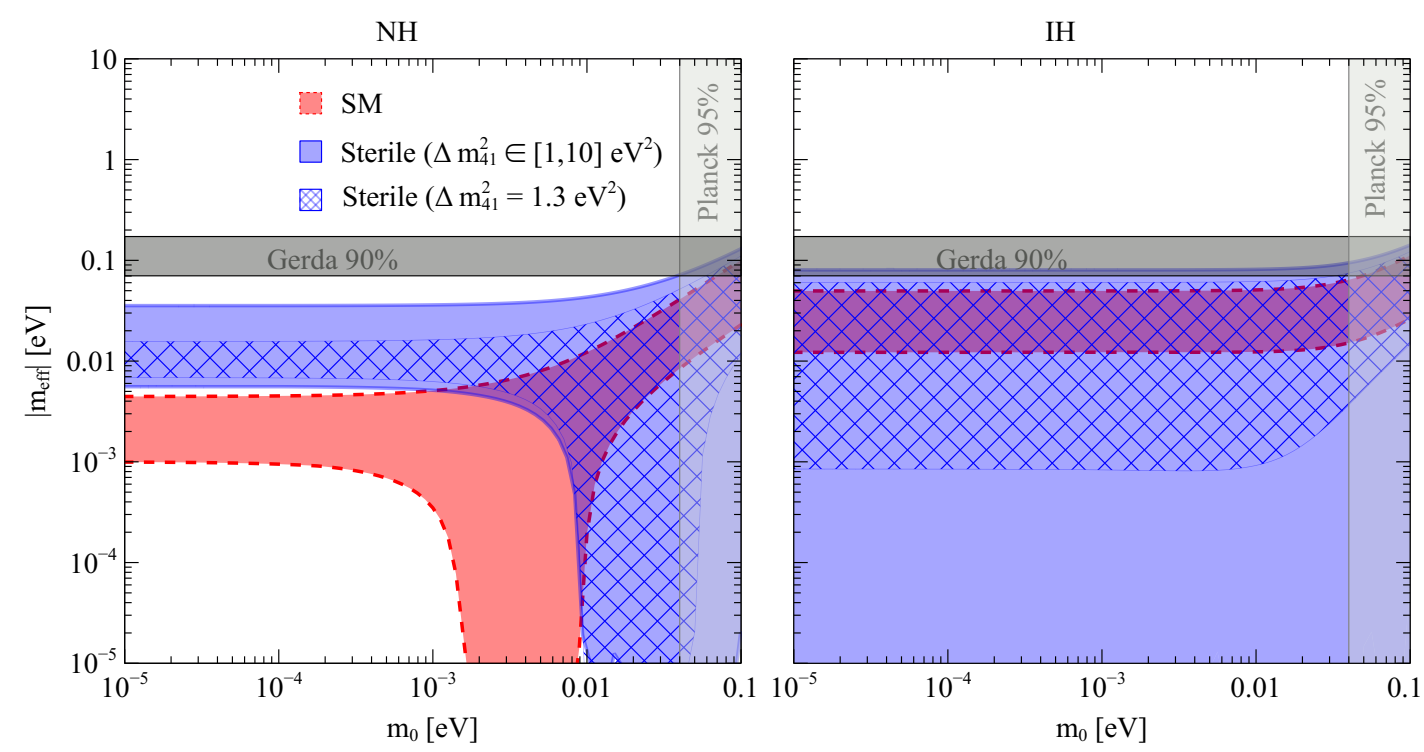

Figure 6. Effective mass of NDBD versus the smallest neutrino mass. The left (right) panel shows the case of $\mathrm{NH}(\mathrm{IH})$. The red (blue) region correspond to $3+0(3+1)$ scenario, labelled as SM (Sterile). The region hatched with blue cross lines represents $\Delta m_{41}^{2}=1.3 \mathrm{eV}^{2}$. The vertical light grey region is excluded by Planck data at 95\% C.L. [102], while the horizontal dark region shows the $90 \%$ sensitivity from GERDA [103].

of light and heavy neutrinos respectively. The values of NME and phase space factor $G_{0 \nu}$ can be find in ref. [104]. The half-life of $0 \nu 2 \beta$ is can generally given as [105]

$$
\frac{1}{T_{1 / 2}}=K_{0 \nu}\left|\Theta_{e j}^{2} \frac{\mu_{j}}{\left\langle p^{2}\right\rangle-\mu_{j}^{2}}\right|^{2},
$$

where $j$ represents the number of light neutrino states and the additional heavy neutrino states. The parameters $\mu_{j}$ and $\Theta_{e j}$ represent the masses of the neutrino states and the mixing with SM neutrinos respectively. In the above, $K_{0 \nu}=G_{0 \nu}\left(\mathcal{M}_{N} m_{p}\right)^{2}$ and $\left\langle p^{2}\right\rangle \equiv-m_{e} m_{p} \frac{\mathcal{M}_{N}}{\mathcal{M}_{\nu}}$. Over the decade, many experiments on improving the lower limits on $T_{1 / 2}^{0 \nu}$ for $0 \nu \beta \beta$ transition have been performed and best stringent bounds have been acquired from germanium-76, Xenon-136 and tellurium-130 [106] isotopes. The experiment GERDA-II, at $90 \%$ C.L. has obtained the corresponding lower limit as $T_{1 / 2}^{0 \nu}>8.0 \times 10^{25}$ year for $G e^{(76)}$ [107], whereas from KamLAND-Zen experiment $X e^{(136)}$, at $90 \%$ C.L. the lower limit on the half-life is obtained as $T_{1 / 2}^{0 \nu}>1.07 \times 10^{26}$ year $[108,109]$.

In presence of a sterile neutrino, the effective mass obtained from $3+0$ framework will be significantly changed. Using eq. (7.1), in figure 6, we show the effective mass for the standard $3+0$ scenario, and for $3+1$ scenario with the variation of the lightest mass. The left and right panels represent $\mathrm{NH}$ and $\mathrm{IH}$, respectively. We consider a variation of $\Delta m_{41}^{2}$ (for $\mathrm{NH}$ ), and $\Delta m_{43}^{2}$ (for $\mathrm{IH}$ ) in between (1-10) $\mathrm{eV}^{2}$. The CP phases $\alpha_{2}, \alpha_{3}$ and $\alpha_{4}$ have been varied in between $-\pi$ to $\pi$. Other oscillation parameters have been varied in their $3 \sigma$ ranges as shown in table 1 [22] as applicable for $\mathrm{NH}$ and $\mathrm{IH}$. Overall we have 
considered $10^{7}$ iterations at each value of the lightest mass in generating figure 6 . The red and blue regions represent the variation of $\left|m_{\mathrm{eff}}\right|$ for the standard $3+0$ scenario, and $3+1$ scenario respectively. As can be seen from the figure the $\left|m_{\mathrm{eff}}\right|$ can be significantly large in the presence of a sterile neutrino with large mass value, and hence constrained from the experimental constraint. To make a connection with our oscillation analyses in the preceding sections, we shade the regions corresponding to $\Delta m_{41}^{2}=1.3 \mathrm{eV}^{2}$ with blue cross lines. Consequently we note a slight shrinking of the blue regions and it largely comes down to below the exclusion limit by Gerda. We note that for $\mathrm{NH}$, there is a complete cancellation of $m_{\mathrm{eff}}$ in the $3+0$ case when the lightest mass approximately lies in the range of $10^{-3}-10^{-2} \mathrm{eV}$. In $3+1$ case, there is no complete cancellation in this region (due to the dominance of the $m_{4}$-term in eq. (7.1) in this range) and it only happens when the lightest mass becomes $10^{-2} \mathrm{eV}$ and beyond. For $\mathrm{IH}$, the standard $3+0$ case shows no total cancellation (dominant $m_{1}$-term in eq. (7.1)), while there is a total cancellation in the $3+1$ case in the range shown. Our results qualitatively agree with [100].

\section{Summary and conclusion}

In this paper we have considered the presence of an $\mathrm{eV}$-scale sterile neutrino (the so called $3+1$ scenario which might turn out to be a possible resolution of the short baseline neutrino oscillation anomalies) and have analyzed how the present and future long baseline experiments T2K, NOvA, DUNE and T2HK can potentially probe the additional CP phases. We discuss how the three CP phases, namely $\delta_{13}, \delta_{24}$ and $\delta_{34}$ can individually affect the oscillation channels under consideration and appear in the probability expression. In light of the constraints on the active-sterile mixing from the global analysis, we estimate how the LBL experiments can probe the parameter spaces associated to the CP phases, by taking a pair of CP phases at a time. Though $\nu_{\mu} \rightarrow \nu_{e}$ oscillation channel contributes the most in probing these parameter spaces, $\nu_{\mu} \rightarrow \nu_{\mu}$ and to a lesser extent $\nu_{\mu} \rightarrow \nu_{\tau}$ channel also help in exploring the $\delta_{24}-\delta_{34}$ parameter space in particularly. By marginalizing over all other parameters we then show how the three individual CP phases can be reconstructed for all possible true values in the whole range of $[-\pi, \pi]$. We find that $\delta_{24}$ and $\delta_{34}$ cannot be reconstructed very efficiently by DUNE and also even after adding data from NOvA and T2K. But adding T2HK data removes much of the degeneracies and the uncertainties in reconstruction become much less. We found that if the active-sterile mixing angles turn out to be lying close to their current upper limits, the enhanced sensitivities to the associated phases make the reconstructions of $\delta_{24}$ and $\delta_{34}$ much better. In contrast the reconstruction of the standard CP phase $\delta_{13}$ is much better even in presence of a light sterile neutrino and this conclusion is almost independent of the size of active-sterile mixing. We then analyze how efficiently the experiments can probe all the parameter spaces associated to one $\mathrm{CP}$ phase and one active-sterile mixing angle. It turns out that the parameter regions connected to the angle $\theta_{14}$ can be probed relatively better that those related to the other two mixing angles. Finally, we briefly show how the relevant parameter spaces in $0 \nu \beta \beta$ get modified in light of the active-sterile constraints used in this analysis. 


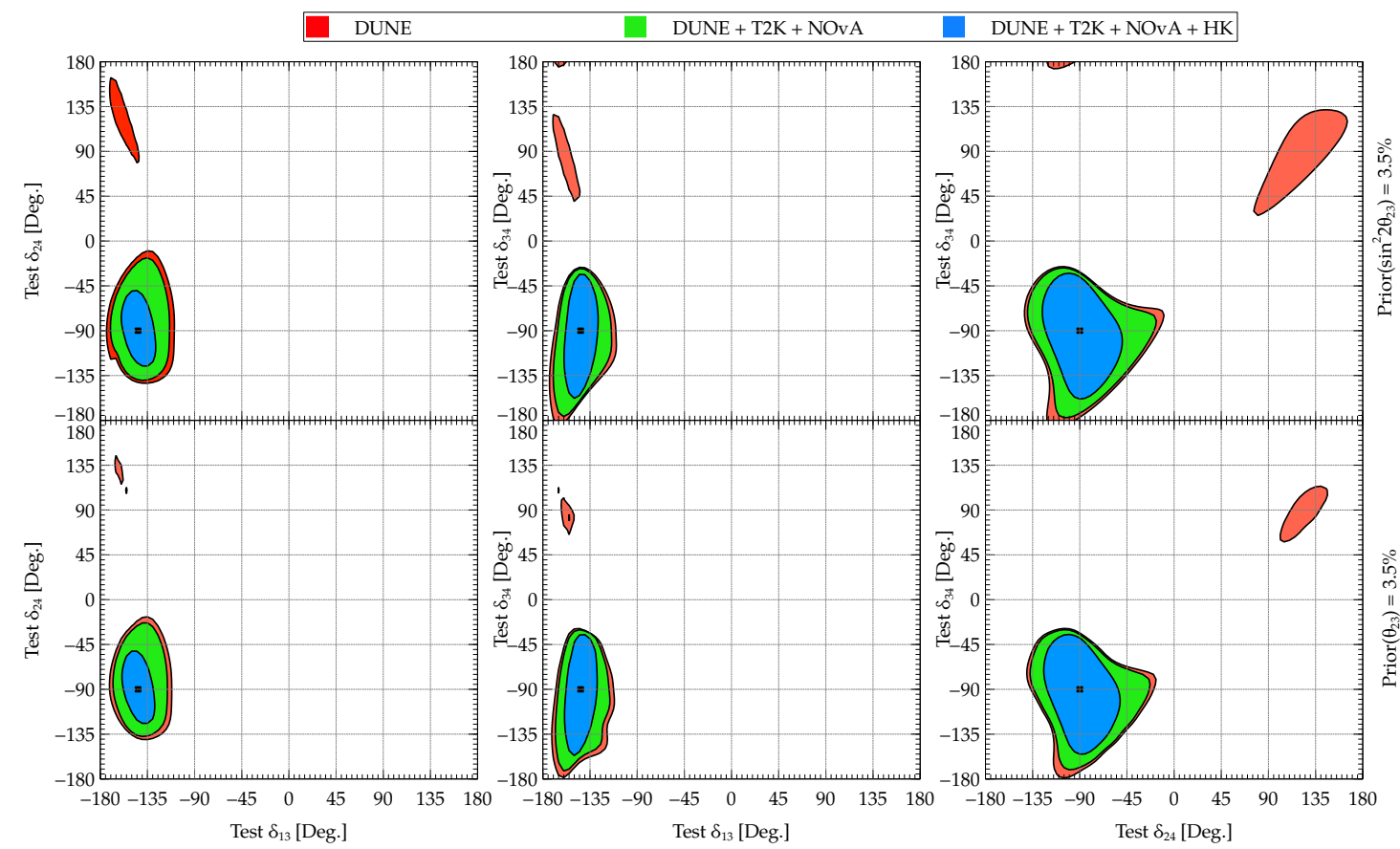

Figure 7. The effect of taking a non-Gaussian prior uncertainty on $\theta_{23}$ on the reconstruction of the CP phases, taken pairwise at a time, at a C.L. of $1 \sigma$ (2 D.O.F.) for DUNE (red), DUNE + $\mathrm{T} 2 \mathrm{~K}+\mathrm{NO} \nu \mathrm{A}$ (green), and $\mathrm{DUNE}+\mathrm{T} 2 \mathrm{~K}+\mathrm{NO} \nu \mathrm{A}+\mathrm{T} 2 \mathrm{HK}$ (blue). The black dots represent the true values to be reconstructed. The top row shows the case of taking a prior uncertainty of $3.5 \%$ on $\sin ^{2} 2 \theta_{23}$. The bottom row (same as that of the bottom row of figure 2), depicts the case of a Gaussian prior uncertainty of $3.5 \%$ on $\theta_{23}$.

\section{Acknowledgments}

N.F. is grateful for a visit to IOP, Bhubaneswar where this project was initialized. N.F. acknowledges the support of Dr. Satyajit Jena of IISER Mohali. M. Masud acknowledges Dr. S.K. Agarwalla of IOP, Bhubaneswar for providing the financial support from the Indian National Science Academy (INSA) Young Scientist Project [INSA/SP/YS/2019/269]. M. Masud is supported by IBS under the project code IBS-R018-D1. M. Mitra acknowledges the financial support from DST INSPIRE Faculty research grant (IFA-14-PH-99), and thanks Indo-French Centre for the Promotion of Advanced Research for the support (grant no: 6304-2). We are grateful to the anonymous referee for constructive suggestions and inputs.

\section{A Effect of $\theta_{23}$-uncertainty}

In all the results so far, we have used a Gaussian prior of $3.5 \%$ on $\theta_{23}$. Since a true value lying in the higher octant of $\theta_{23}$ has been used in the analyses, we also need to study the effect of using a non-Gaussian prior in order to properly consider both the octants when we marginalise over $\theta_{23}$. For this purpose, we take a prior uncertainty of $3.5 \%$ on $\sin ^{2} 2 \theta_{23}$ in the reconstruction of the $\mathrm{CP}$ phases and compare it with the case of considering a Gaussian prior in figure 7 . We note that, taking the prior on $\sin ^{2} 2 \theta_{23}$ results in only a mild spread 

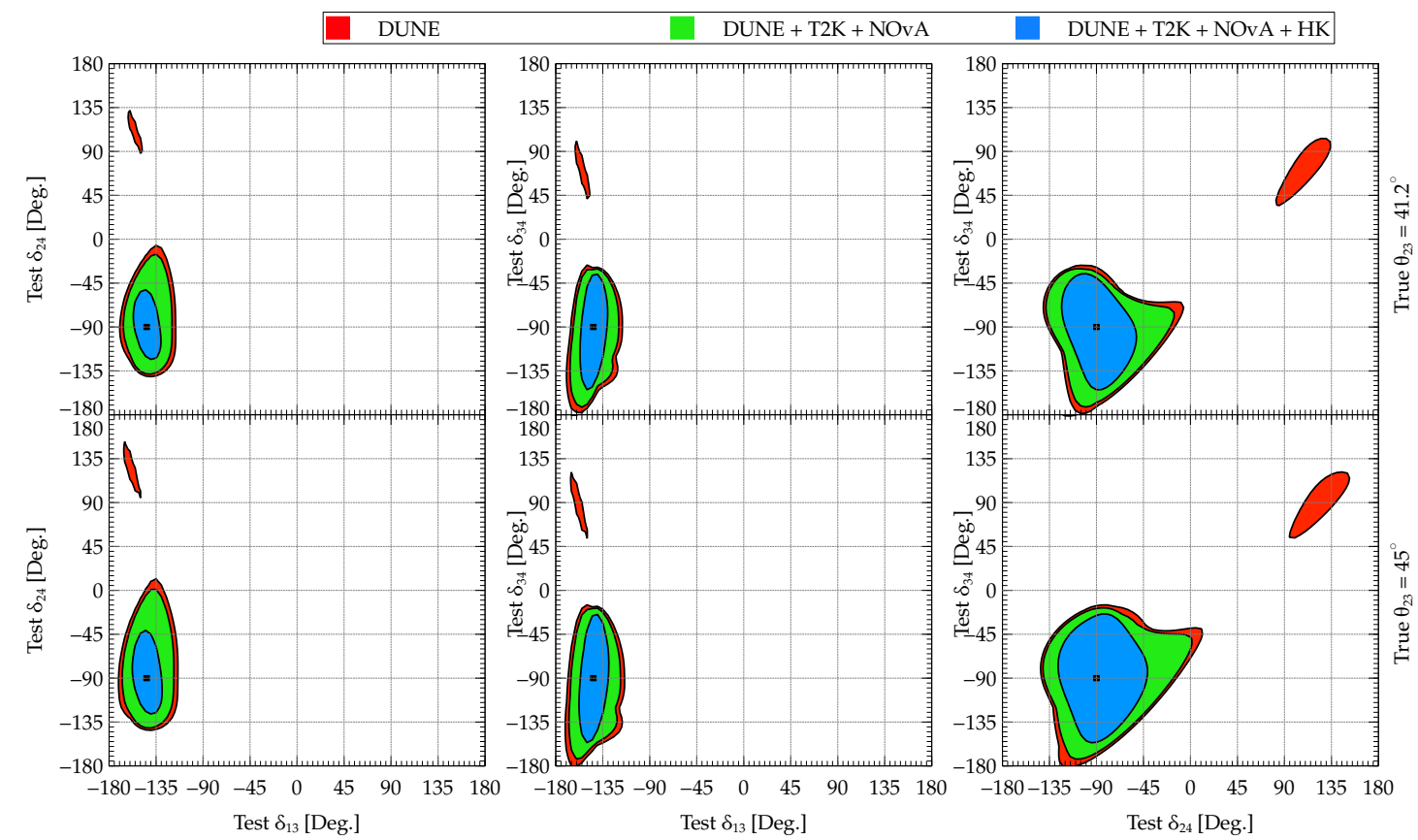

Figure 8. Effect of considering different true $\theta_{23}$-octant while probing the $\mathrm{CP}$ phases, taken pairwise at a time (same as the bottom row of figure 7 , but for lower octant and maximal mixing for $\left.\theta_{23}\right)$.

in the contours, especially along the axes of the sterile $\mathrm{CP}$ phases $\delta_{24}$ or $\delta_{34}$. But this does not change our overall results qualitatively. For e.g., though the contours showing the degenerate solutions at DUNE (red) gets bigger with a prior on $\sin ^{2} 2 \theta_{23}$, addition of data from other experiments still removes this degenerate fake solution as before.

\section{B Effect of different true choice of the standard parameters}

The best fit values of the standard oscillation parameters used in our analyses (as mentioned in table 1) were obtained under the $3+0$ scenario. It has been shown in literature that some of these standard parameters are prone to significant changes in the presence of a light sterile neutrino. For e.g., the values of $\theta_{23}, \Delta m_{31}^{2}, \delta_{13}$ can change so much that the issues of correct octant, mass hierarchy or CP Violation become very ambiguous (see for e.g., $[63,64])$. The reactor mixing angle $\theta_{13}$, on the other hand is quite robust even in the $3+1$ scenario [110]. Though the marginalisation process during $\Delta \chi^{2}$ calculation partially takes care of the uncertainties of relevant parameters in the fit, a brief discussion regarding the effect of different true values of the standard parameters are in order. In figure 3 , we had already illustrated our results for all possible true values of the $\mathrm{CP}$ violating phases. Here we now show in figure 8, the impact of the true choice of lower octant (top row) and maximal mixing (bottom row) of $\theta_{23}$ in probing the CP phases. This is similar to the bottom row of figure 7 , but for two different possible true choices of $\theta_{23}$. We see that the contours remain qualitatively similar when different true octants of $\theta_{23}$ are considered. For 

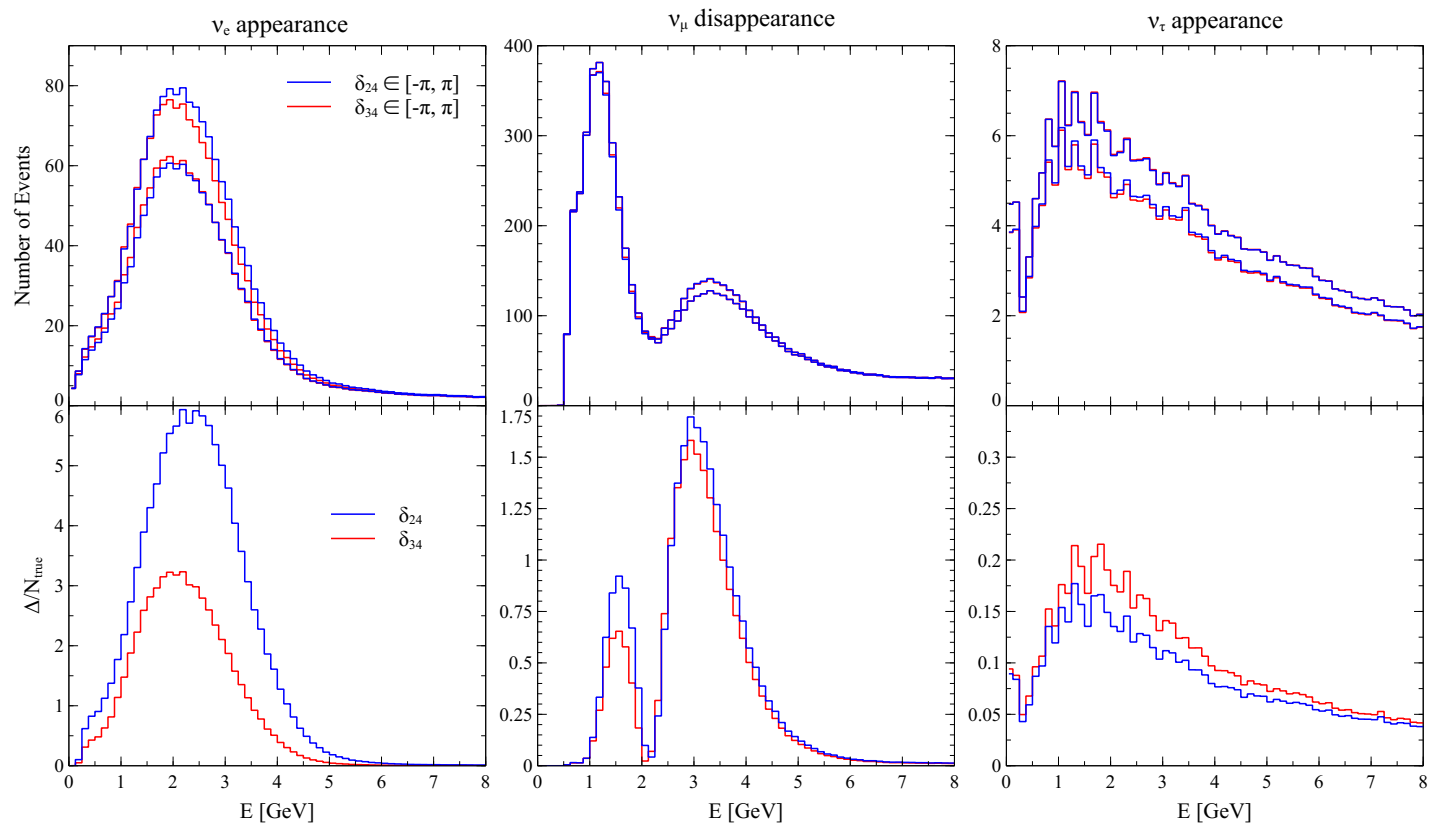

Figure 9. Top row shows the bands for event spectra at DUNE due to variations of $3+1 \mathrm{CP}$ phases for $\nu_{\mu} \rightarrow \nu_{e}, \nu_{\mu} \rightarrow \nu_{\mu}$ and $\nu_{\mu} \rightarrow \nu_{\tau}$ channels respectively. The band within blue (red) lines corresponds to the variation of $\delta_{24}\left(\delta_{34}\right)$ in the range $[-\pi, \pi]$. The bottom row shows the corresponding spread $\Delta$ (square of difference between the two same coloured curves in the top row) over $N_{\text {true }}$ (number of events when $\delta_{24}, \delta_{34}$ are fixed at zero). See text for details.

the case of maximal mixing, a slight elongation of the contours are observed, especially along the $\delta_{24}$ and $\delta_{34}$ directions. We have also checked that for inverted hierarchy (IH) of neutrino masses our results remain similar. This is due to the fact that even in presence of a sterile neutrino, the mass hierarchy sensitivity of DUNE, though deteriorates, remains high enough $(\gtrsim 5 \sigma)[64]$.

\section{Understanding the role of channels to probe $\delta_{24}$ and $\delta_{34}$}

Here we make an attempt to understand the relative role of the three oscillation channels to probe the sterile $\mathrm{CP}$ phases $\delta_{24}$ and $\delta_{34}$ as observed in figure 4 . For a simpler and more intuitive explanation we use the following Gaussian $\Delta \chi^{2}$ (the Poissonian definition in eq. (3.1) reduces to the Gaussian version for sufficiently large number of events [3].):

$$
\Delta \chi^{2} \sim \operatorname{Min}_{\text {test }} \sum_{\text {bin }}\left[\frac{\left(N_{\mu e}^{\text {true }}-N_{\mu e}^{\text {test }}\right)^{2}}{N_{\mu e}^{\text {true }}}+\frac{\left(N_{\mu \mu}^{\text {true }}-N_{\mu \mu}^{\text {test }}\right)^{2}}{N_{\mu \mu}^{\text {true }}}+\frac{\left(N_{\mu \tau}^{\text {true }}-N_{\mu \tau}^{\text {test }}\right)^{2}}{N_{\mu \tau}^{\text {true }}}\right]+(\text { prior \& systematics }) .
$$

Here $N_{\alpha \beta}^{\text {true }}\left(N_{\alpha \beta}^{\text {test }}\right)$ is the true (test) set of events coming from the $\nu_{\alpha} \rightarrow \nu_{\beta}$ oscillation channel. Note that, we have sketched here only the relevant statistical part of the $\Delta \chi^{2}$ and ignored the prior and systematics. In order to understand figure 4 , we first estimate the band of $N_{\alpha \beta}^{\text {test }}$ corresponding to individual variations of $\delta_{24} \in[-\pi, \pi]$ and $\delta_{34} \in[-\pi, \pi]$. These spectra are illustrated for the three channels in figure 9 (top row). The $\Delta \chi^{2}$ for reconstructing $\delta_{24}$ and $\delta_{34}$ is governed by the spread/width of such event bands and we 

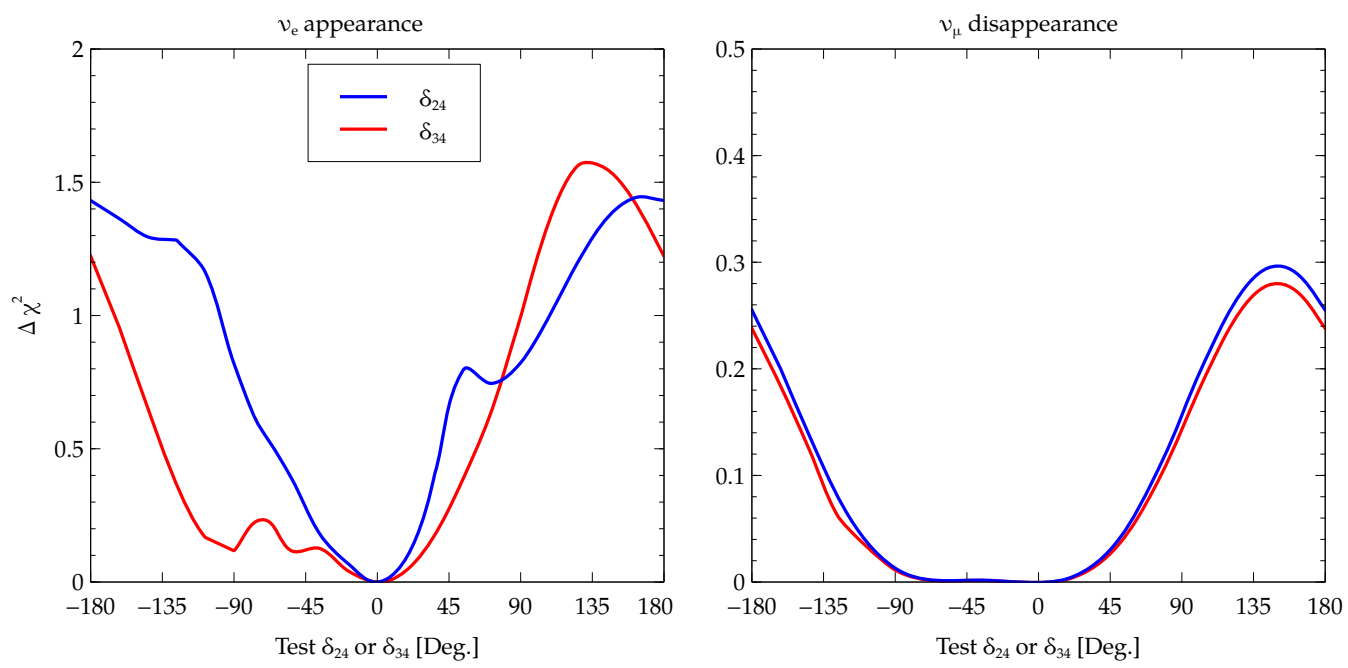

Figure 10. $\Delta \chi^{2}$ in probing $\delta_{24}$ (blue) or $\delta_{34}$ (red) for $\nu_{\mu} \rightarrow \nu_{e}$ (left) and $\nu_{\mu} \rightarrow \nu_{\mu}$ (right) channels. The true values of $\delta_{24}$ or $\delta_{34}$ are considered to be zero while the test values are shown along the horizontal axes.

calculate the square of the vertical width at each energy bin and refer it by $\Delta$. In accordance with figure 4 we then generate $N_{\alpha \beta}^{\text {true }}$ corresponding to $\delta_{24}=0$ and $\delta_{34}=0$. Now, following eq. (C.1), we plot $\Delta / N_{\alpha \beta}^{\text {true }}$ for the three channels in the bottom row of figure 9 , both for $\delta_{24}$ (blue) and $\delta_{34}$ (red). This approximately gives us an idea of the relative contribution of the channels in the reconstruction of the sterile CP phases. As expected, the $\nu_{\mu} \rightarrow \nu_{e}$ channel gives the dominant contribution and $\delta_{24}$-reconstruction is expected to be slightly better than $\delta_{34} . \nu_{\mu} \rightarrow \nu_{\mu}$ channel also plays a role and this mainly comes due to the large number of $\nu_{\mu}$ events. Finally $\nu_{\mu} \rightarrow \nu_{\tau}$ channel has a small but non-negligible contribution. Both $\nu_{\mu} \rightarrow \nu_{\mu}$ and $\nu_{\mu} \rightarrow \nu_{\tau}$ channels provide similar capability of reconstructing $\delta_{24}$ and $\delta_{34}$.

However, since the $\nu_{\mu} \rightarrow \nu_{\mu}$ channel events are also limited by systematic uncertainties, some remarks regarding the effect of systematics in eq. (C.1) are in order. Extending our analysis in this appendix from the level of events to that of $\Delta \chi^{2}$, we show in figure 10, a comparison between the $\nu_{\mu} \rightarrow \nu_{e}$ and $\nu_{\mu} \rightarrow \nu_{\mu}$ channels in probing $\delta_{24}$ or $\delta_{34}$. As in figure 9 , here we also consider the true values of $\delta_{24}$ or $\delta_{34}$ to be 0 and then plot the $\Delta \chi^{2}$ as a function of the test values of $\delta_{24}$ (blue) or $\delta_{34}$ (red), after marginalising over all other relevant test parameters. The left (right) panel shows the contribution of the $\nu_{\mu} \rightarrow \nu_{e}\left(\nu_{\mu} \rightarrow \nu_{\mu}\right)$ channel alone. As mentioned in section 3, 2\% (5\%) systematics has been considered in the $\nu_{\mu} \rightarrow \nu_{e}\left(\nu_{\mu} \rightarrow \nu_{\mu}\right)$ signal, following [91] (along with various background systematics as well). Due to this larger systematic uncertainty, we note from figure 10, that the relative role of $\nu_{\mu} \rightarrow \nu_{\mu}$ channel in probing the sterile phases with respect to $\nu_{\mu} \rightarrow \nu_{e}$ channel further diminishes compared to that of figure 9. Nevertheless, even with such systematics the contribution of the $\nu_{\mu} \rightarrow \nu_{\mu}$ channel can still be roughly $\sim 15 \%-20 \%$ of that of $\nu_{\mu} \rightarrow \nu_{e}$ in the favourable region of the parameter space. Thus when all the oscillation channels are included to do a combined $\Delta \chi^{2}$ analysis, the interplay and complementarity among the channels improves the sensitivity to probe the CP phases. These findings are consistent with the full statistical analysis as discussed in section 5 . 
Open Access. This article is distributed under the terms of the Creative Commons Attribution License (CC-BY 4.0), which permits any use, distribution and reproduction in any medium, provided the original author(s) and source are credited.

\section{References}

[1] Super-Kamiokande collaboration, Evidence for oscillation of atmospheric neutrinos, Phys. Rev. Lett. 81 (1998) 1562 [hep-ex/9807003] [INSPIRE].

[2] SNO collaboration, Direct evidence for neutrino flavor transformation from neutral current interactions in the Sudbury Neutrino Observatory, Phys. Rev. Lett. 89 (2002) 011301 [nucl-ex/0204008] [INSPIRE].

[3] Particle Data Group collaboration, Review of Particle Physics, PTEP 2020 (2020) 083C01 [INSPIRE].

[4] A.D. Sakharov, Violation of CP Invariance, $C$ asymmetry, and baryon asymmetry of the universe, Pisma Zh. Eksp. Teor. Fiz. 5 (1967) 32 [Sov. Phys. Usp. 34 (1991) 392] [INSPIRE].

[5] M. Fukugita and T. Yanagida, Baryogenesis Without Grand Unification, Phys. Lett. B 174 (1986) 45 [INSPIRE].

[6] R.N. Mohapatra and G. Senjanović, Neutrino Mass and Spontaneous Parity Nonconservation, Phys. Rev. Lett. 44 (1980) 912 [INSPIRE].

[7] J. Schechter and J.W.F. Valle, Neutrino Masses in $\mathrm{SU}(2) \times \mathrm{U}(1)$ Theories, Phys. Rev. D 22 (1980) 2227 [INSPIRE].

[8] S.T. Petcov, On PseudoDirac Neutrinos, Neutrino Oscillations and Neutrinoless Double beta Decay, Phys. Lett. B 110 (1982) 245 [inSPIRE].

[9] W.C. Haxton and G.J. Stephenson, Double beta Decay, Prog. Part. Nucl. Phys. 12 (1984) 409 [INSPIRE].

[10] C.S. Lam, A 2-3 symmetry in neutrino oscillations, Phys. Lett. B 507 (2001) 214 [hep-ph/0104116] [INSPIRE].

[11] P.F. Harrison and W.G. Scott, $\mu-\tau$ reflection symmetry in lepton mixing and neutrino oscillations, Phys. Lett. B 547 (2002) 219 [hep-ph/0210197] [INSPIRE].

[12] T2K collaboration, Observation of Electron Neutrino Appearance in a Muon Neutrino Beam, Phys. Rev. Lett. 112 (2014) 061802 [arXiv:1311.4750] [InSPIRE].

[13] NOvA collaboration, NOvA: Proposal to Build a 30 Kiloton Off-Axis Detector to Study $\nu_{\mu} \rightarrow \nu_{e}$ Oscillations in the NuMI Beamline, hep-ex/0503053 [INSPIRE].

[14] T2K collaboration, Constraint on the matter-antimatter symmetry-violating phase in neutrino oscillations, Nature 580 (2020) 339 [Erratum ibid. 583 (2020) E16] [arXiv: 1910.03887] [INSPIRE].

[15] NOvA collaboration, First Measurement of Neutrino Oscillation Parameters using Neutrinos and Antineutrinos by NOvA, Phys. Rev. Lett. 123 (2019) 151803 [arXiv: 1906.04907] [INSPIRE].

[16] DUNE collaboration, Long-Baseline Neutrino Facility (LBNF) and Deep Underground Neutrino Experiment (DUNE): Conceptual Design Report, Volume 2: The Physics Program for DUNE at LBNF, arXiv:1512.06148 [INSPIRE]. 
[17] DUNE collaboration, Deep Underground Neutrino Experiment (DUNE), Far Detector Technical Design Report, Volume II: DUNE Physics, arXiv:2002.03005 [INSPIRE].

[18] DUNE collaboration, Long-baseline neutrino oscillation physics potential of the DUNE experiment, Eur. Phys. J. C 80 (2020) 978 [arXiv: 2006.16043] [INSPIRE].

[19] Hyper-Kamiokande Proto- collaboration, Physics potential of a long-baseline neutrino oscillation experiment using a J-PARC neutrino beam and Hyper-Kamiokande, PTEP 2015 (2015) 053C02 [arXiv:1502.05199] [InSPIRE].

[20] Hyper-Kamiokande collaboration, Physics potentials with the second Hyper-Kamiokande detector in Korea, PTEP 2018 (2018) 063C01 [arXiv:1611.06118] [INSPIRE].

[21] ESSNuSB collaboration, A very intense neutrino super beam experiment for leptonic CP-violation discovery based on the European spallation source linac, Nucl. Phys. B $\mathbf{8 8 5}$ (2014) 127 [arXiv: 1309.7022] [INSPIRE].

[22] P.F. de Salas et al., 2020 global reassessment of the neutrino oscillation picture, JHEP 02 (2021) 071 [arXiv : 2006.11237] [inSPIRE].

[23] Valencia-Globalfit, Neutrino Global Fit, (2020) http://globalfit.astroparticles.es.

[24] F. Capozzi, E. Lisi, A. Marrone and A. Palazzo, Current unknowns in the three neutrino framework, Prog. Part. Nucl. Phys. 102 (2018) 48 [arXiv:1804.09678] [InSPIRE].

[25] I. Esteban, M.C. Gonzalez-Garcia, M. Maltoni, T. Schwetz and A. Zhou, The fate of hints: updated global analysis of three-flavor neutrino oscillations, JHEP 09 (2020) 178 [arXiv: 2007.14792] [INSPIRE].

[26] LSND collaboration, Evidence for neutrino oscillations from the observation of $\bar{\nu}_{e}$ appearance in a $\bar{\nu}_{\mu}$ beam, Phys. Rev. D 64 (2001) 112007 [hep-ex/0104049] [INSPIRE].

[27] MiniBooNE collaboration, Unexplained Excess of Electron-Like Events From a $1 \mathrm{GeV}$ Neutrino Beam, Phys. Rev. Lett. 102 (2009) 101802 [arXiv:0812.2243] [INSPIRE].

[28] T.A. Mueller et al., Improved Predictions of Reactor Antineutrino Spectra, Phys. Rev. C 83 (2011) 054615 [arXiv:1101.2663] [INSPIRE].

[29] G. Mention et al., The Reactor Antineutrino Anomaly, Phys. Rev. D 83 (2011) 073006 [arXiv:1101.2755] [INSPIRE].

[30] P. Huber, On the determination of anti-neutrino spectra from nuclear reactors, Phys. Rev. C 84 (2011) 024617 [Erratum ibid. 85 (2012) 029901] [arXiv: 1106.0687] [INSPIRE].

[31] ALEPH collaboration, Determination of the Number of Light Neutrino Species, Phys. Lett. B 231 (1989) 519 [INSPIRE].

[32] MiniBoonE collaboration, Significant Excess of ElectronLike Events in the MiniBooNE Short-Baseline Neutrino Experiment, Phys. Rev. Lett. 121 (2018) 221801 [arXiv: 1805.12028] [INSPIRE].

[33] MiniBooNE collaboration, Updated MiniBooNE neutrino oscillation results with increased data and new background studies, Phys. Rev. D 103 (2021) 052002 [arXiv:2006.16883] [INSPIRE].

[34] ICECUBE collaboration, Searching for eV-scale sterile neutrinos with eight years of atmospheric neutrinos at the IceCube Neutrino Telescope, Phys. Rev. D 102 (2020) 052009 [arXiv: 2005.12943] [INSPIRE]. 
[35] KATRIN collaboration, KATRIN: A Next generation tritium beta decay experiment with sub-eV sensitivity for the electron neutrino mass. Letter of intent, hep-ex/0109033 [INSPIRE].

[36] MicroBoone, LAr1-ND and ICARUS-WA104 collaborations, A Proposal for a Three Detector Short-Baseline Neutrino Oscillation Program in the Fermilab Booster Neutrino Beam, arXiv:1503.01520 [INSPIRE].

[37] ANTARES collaboration, ANTARES: the first undersea neutrino telescope, Nucl. Instrum. Meth. A 656 (2011) 11 [arXiv:1104.1607] [INSPIRE].

[38] NEOS collaboration, Sterile Neutrino Search at the NEOS Experiment, Phys. Rev. Lett. 118 (2017) 121802 [arXiv:1610.05134] [INSPIRE].

[39] SoLid collaboration, A novel segmented-scintillator antineutrino detector, 2017 JINST 12 P04024 [arXiv: 1703.01683] [INSPIRE].

[40] NEUTRINO-4 collaboration, First Observation of the Oscillation Effect in the Neutrino-4 Experiment on the Search for the Sterile Neutrino, Pisma Zh. Eksp. Teor. Fiz. 109 (2019) 209 [arXiv: 1809.10561] [INSPIRE].

[41] PROSPECT collaboration, First search for short-baseline neutrino oscillations at HFIR with PROSPECT, Phys. Rev. Lett. 121 (2018) 251802 [arXiv: 1806. 02784] [InSPIRE].

[42] STEREO collaboration, The STEREO Experiment, 2018 JINST 13 P07009 [arXiv: 1804.09052] [INSPIRE].

[43] STEREO collaboration, Improved sterile neutrino constraints from the STEREO experiment with 179 days of reactor-on data, Phys. Rev. D 102 (2020) 052002 [arXiv: 1912.06582] [INSPIRE].

[44] DANSS collaboration, Search for sterile neutrinos at the DANSS experiment, Phys. Lett. B 787 (2018) 56 [arXiv:1804.04046] [INSPIRE].

[45] JSNS2 collaboration, Status of JSNS $-J$-PARC Sterile Neutrino Search at J-PARC Spallation Neutron Source, J. Phys. Conf. Ser. 1468 (2020) 012176 [INSPIRE].

[46] JSNS2 collaboration, JSNS Experiment, talk at Neutrino2020, June, 2020 https://indico.fnal.gov/event/43209/contributions/187854/attachments/129166/159526/ Neutrino2020_JSNS2_v3.pdf.

[47] A.P. Serebrov and R.M. Samoilov, Analysis of the Results of the Neutrino-4 Experiment on the Search for the Sterile Neutrino and Comparison with Results of Other Experiments, JETP Lett. 112 (2020) 199 [Pisma Zh. Eksp. Teor. Fiz. 112 (2020) 211] [arXiv: 2003. 03199] [INSPIRE].

[48] A.P. Serebrov et al., Preparation of the Neutrino-4 experiment on search for sterile neutrino and the obtained results of measurements, Phys. Rev. D 104 (2021) 032003 [arXiv: 2005. 05301] [INSPIRE].

[49] Neutrino-4 collaboration, Observation of sterile antineutrino oscillation in Neutrino-4 experiment at SM-3 reactor, talk at Neutrino2020, June, 2020 https://indico.fnal.gov/event/43209/contributions/187878/attachments/129237/ Serebrov_Neutrino-4_25july.pdf.

[50] C. Giunti, Statistical Significance of Reactor Antineutrino Active-Sterile Oscillations, Phys. Rev. D 101 (2020) 095025 [arXiv: 2004.07577] [INSPIRE]. 
[51] P. Coloma, P. Huber and T. Schwetz, Statistical interpretation of sterile neutrino oscillation searches at reactors, Eur. Phys. J. C 81 (2021) 2 [arXiv:2008.06083] [INSPIRE].

[52] C. Giunti, Y.F. Li, C.A. Ternes and Y.Y. Zhang, Neutrino-4 anomaly: oscillations or fluctuations?, Phys. Lett. B 816 (2021) 136214 [arXiv:2101.06785] [INSPIRE].

[53] ANTARES collaboration, Measuring the atmospheric neutrino oscillation parameters and constraining the $3+1$ neutrino model with ten years of ANTARES data, JHEP 06 (2019) 113 [arXiv: 1812.08650] [INSPIRE].

[54] COHERENT collaboration, Observation of Coherent Elastic Neutrino-Nucleus Scattering, Science 357 (2017) 1123 [arXiv: 1708.01294] [INSPIRE].

[55] O.G. Miranda, D.K. Papoulias, O. Sanders, M. Tórtola and J.W.F. Valle, Future CEvNS experiments as probes of lepton unitarity and light-sterile neutrinos, Phys. Rev. D 102 (2020) 113014 [arXiv: 2008. 02759] [inSPIRE].

[56] R. Gandhi, B. Kayser, M. Masud and S. Prakash, The impact of sterile neutrinos on CP measurements at long baselines, JHEP 11 (2015) 039 [arXiv:1508.06275] [INSPIRE].

[57] S. Böser et al., Status of Light Sterile Neutrino Searches, Prog. Part. Nucl. Phys. 111 (2020) 103736 [arXiv: 1906.01739] [inSPIRE].

[58] N. Klop and A. Palazzo, Imprints of CP-violation induced by sterile neutrinos in T2K data, Phys. Rev. D 91 (2015) 073017 [arXiv:1412.7524] [InSPIRE].

[59] J.M. Berryman, A. de Gouvêa, K.J. Kelly and A. Kobach, Sterile neutrino at the Deep Underground Neutrino Experiment, Phys. Rev. D 92 (2015) 073012 [arXiv:1507.03986] [INSPIRE].

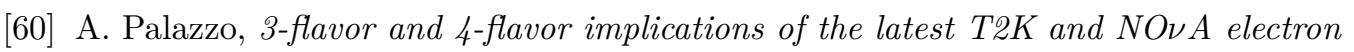
(anti-)neutrino appearance results, Phys. Lett. B 757 (2016) 142 [arXiv:1509.03148] [INSPIRE].

[61] S.K. Agarwalla, S.S. Chatterjee, A. Dasgupta and A. Palazzo, Discovery Potential of T2K and NOvA in the Presence of a Light Sterile Neutrino, JHEP 02 (2016) 111 [arXiv: 1601.05995] [INSPIRE].

[62] S.K. Agarwalla, S.S. Chatterjee and A. Palazzo, Physics Reach of DUNE with a Light Sterile Neutrino, JHEP 09 (2016) 016 [arXiv: 1603. 03759] [inSPIRE].

[63] S.K. Agarwalla, S.S. Chatterjee and A. Palazzo, Octant of $\theta_{23}$ in danger with a light sterile neutrino, Phys. Rev. Lett. 118 (2017) 031804 [arXiv:1605. 04299] [INSPIRE].

[64] D. Dutta, R. Gandhi, B. Kayser, M. Masud and S. Prakash, Capabilities of long-baseline experiments in the presence of a sterile neutrino, JHEP 11 (2016) 122 [arXiv:1607.02152] [INSPIRE].

[65] J. Rout, M. Masud and P. Mehta, Can we probe intrinsic CP and T violations and nonunitarity at long baseline accelerator experiments?, Phys. Rev. D 95 (2017) 075035 [arXiv: 1702.02163] [INSPIRE].

[66] K.J. Kelly, Searches for new physics at the Hyper-Kamiokande experiment, Phys. Rev. D 95 (2017) 115009 [arXiv: 1703.00448] [INSPIRE].

[67] M. Ghosh, S. Gupta, Z.M. Matthews, P. Sharma and A.G. Williams, Study of parameter

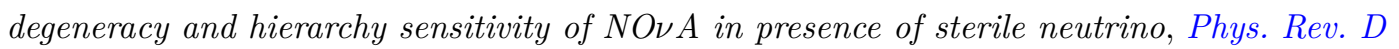
96 (2017) 075018 [arXiv: 1704.04771] [INSPIRE]. 
[68] S. Choubey, D. Dutta and D. Pramanik, Imprints of a light Sterile Neutrino at DUNE, T2HK and T2HKK, Phys. Rev. D 96 (2017) 056026 [arXiv:1704.07269] [inSPIRE].

[69] P. Coloma, D.V. Forero and S.J. Parke, DUNE Sensitivities to the Mixing between Sterile and Tau Neutrinos, JHEP 07 (2018) 079 [arXiv: 1707.05348] [INSPIRE].

[70] J. Tang, Y. Zhang and Y.-F. Li, Probing Direct and Indirect Unitarity Violation in Future Accelerator Neutrino Facilities, Phys. Lett. B 774 (2017) 217 [arXiv:1708. 04909] [INSPIRE].

[71] S. Choubey, D. Dutta and D. Pramanik, Measuring the Sterile Neutrino CP Phase at DUNE and T2HK, Eur. Phys. J. C 78 (2018) 339 [arXiv:1711.07464] [inSPIRE].

[72] S.K. Agarwalla, S.S. Chatterjee and A. Palazzo, Signatures of a Light Sterile Neutrino in T2HK, JHEP 04 (2018) 091 [arXiv: 1801.04855] [INSPIRE].

[73] S. Gupta, Z.M. Matthews, P. Sharma and A.G. Williams, The Effect of a Light Sterile

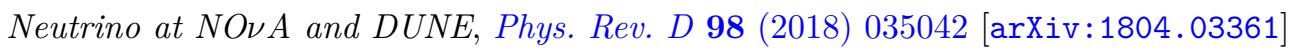
[INSPIRE].

[74] S. Choubey, D. Dutta and D. Pramanik, Exploring fake solutions in the sterile neutrino sector at long-baseline experiments, Eur. Phys. J. C 79 (2019) 968 [arXiv:1811.08684] [INSPIRE].

[75] A. De Gouvêa, K.J. Kelly, G.V. Stenico and P. Pasquini, Physics with Beam Tau-Neutrino Appearance at DUNE, Phys. Rev. D 100 (2019) 016004 [arXiv: 1904.07265] [INSPIRE].

[76] A. Ghoshal, A. Giarnetti and D. Meloni, On the role of the $\nu_{\tau}$ appearance in DUNE in constraining standard neutrino physics and beyond, JHEP 12 (2019) 126 [arXiv: 1906. 06212] [INSPIRE].

[77] S. Kumar Agarwalla, S.S. Chatterjee and A. Palazzo, Physics potential of ESSLSB in the presence of a light sterile neutrino, JHEP 12 (2019) 174 [arXiv:1909.13746] [INSPIRE].

[78] R. Majhi, C. Soumya and R. Mohanta, Light sterile neutrinos and their implications on currently running long-baseline and neutrinoless double beta decay experiments, J. Phys. G 47 (2020) 095002 [arXiv: 1911.10952] [INSPIRE].

[79] M. Ghosh, T. Ohlsson and S. Rosauro-Alcaraz, Sensitivity to light sterile neutrinos at ESSnuSB, JHEP 03 (2020) 026 [arXiv:1912.10010] [INSPIRE].

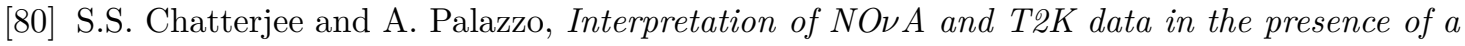
light sterile neutrino, arXiv:2005.10338 [INSPIRE].

[81] C. Giunti and T. Lasserre, eV-scale Sterile Neutrinos, Ann. Rev. Nucl. Part. Sci. 69 (2019) 163 [arXiv: 1901.08330] [INSPIRE].

[82] A. Diaz, C.A. Argüelles, G.H. Collin, J.M. Conrad and M.H. Shaevitz, Where Are We With Light Sterile Neutrinos?, Phys. Rept. 884 (2020) 1 [arXiv: 1906.00045] [InSPIRE].

[83] A. Palazzo, Exploring Light Sterile Neutrinos at Long Baseline Experiments: A Review, Universe 6 (2020) 41 [INSPIRE].

[84] A. Chatla and B.A. Bambah, Effect of Sterile Phases on Degeneracy Resolution Capabilities of LBL Experiments, Phys. At. Nucl. 84 (2021) 377 [arXiv: 2010.06321] [INSPIRE].

[85] M. Dentler et al., Updated Global Analysis of Neutrino Oscillations in the Presence of eV-Scale Sterile Neutrinos, JHEP 08 (2018) 010 [arXiv: 1803.10661] [INSPIRE]. 
[86] B. Yue, W. Li, J. Ling and F. Xu, A compact analytical approximation for a light sterile neutrino oscillation in matter, Chin. Phys. C 44 (2020) 103001 [arXiv:1906.03781] [INSPIRE].

[87] P. Huber, M. Lindner and W. Winter, Simulation of long-baseline neutrino oscillation experiments with GLoBES (General Long Baseline Experiment Simulator), Comput. Phys. Commun. 167 (2005) 195 [hep-ph/0407333] [INSPIRE].

[88] P. Huber, J. Kopp, M. Lindner, M. Rolinec and W. Winter, New features in the simulation of neutrino oscillation experiments with GLoBES 3.0: General Long Baseline Experiment Simulator, Comput. Phys. Commun. 177 (2007) 432 [hep-ph/0701187] [InSPIRE].

[89] J. Kopp, Efficient numerical diagonalization of hermitian $3 \times 3$ matrices, Int. J. Mod. Phys. C 19 (2008) 523 [physics/0610206] [rNSPIRE].

[90] J. Kopp, M. Lindner, T. Ota and J. Sato, Non-standard neutrino interactions in reactor and superbeam experiments, Phys. Rev. D 77 (2008) 013007 [arXiv:0708.0152] [INSPIRE].

[91] DUNE collaboration, Experiment Simulation Configurations Used in DUNE CDR, arXiv: 1606.09550 [INSPIRE].

[92] P. Machado, H. Schulz and J. Turner, Tau neutrinos at DUNE: New strategies, new opportunities, Phys. Rev. D 102 (2020) 053010 [arXiv:2007.00015] [InSPIRE].

[93] T2K collaboration, The T2K Experiment, Nucl. Instrum. Meth. A 659 (2011) 106 [arXiv:1106.1238] [INSPIRE].

[94] Hyper-Kamiokande collaboration, Hyper-Kamiokande Design Report, arXiv:1805.04163 [INSPIRE].

[95] P. Huber, M. Lindner and W. Winter, Superbeams versus neutrino factories, Nucl. Phys. B 645 (2002) 3 [hep-ph/0204352] [INSPIRE].

[96] G.L. Fogli, E. Lisi, A. Marrone, D. Montanino and A. Palazzo, Getting the most from the statistical analysis of solar neutrino oscillations, Phys. Rev. D 66 (2002) 053010 [hep-ph/0206162] [INSPIRE].

[97] M.C. Gonzalez-Garcia and M. Maltoni, Atmospheric neutrino oscillations and new physics, Phys. Rev. D 70 (2004) 033010 [hep-ph/0404085] [INSPIRE].

[98] R. Gandhi, P. Ghoshal, S. Goswami, P. Mehta, S.U. Sankar and S. Shalgar, Mass Hierarchy Determination via future Atmospheric Neutrino Detectors, Phys. Rev. D 76 (2007) 073012 [arXiv: 0707.1723] [INSPIRE].

[99] X. Qian, A. Tan, W. Wang, J.J. Ling, R.D. McKeown and C. Zhang, Statistical Evaluation of Experimental Determinations of Neutrino Mass Hierarchy, Phys. Rev. D 86 (2012) 113011 [arXiv: 1210.3651] [INSPIRE].

[100] C. Giunti and E.M. Zavanin, Predictions for Neutrinoless Double-Beta Decay in the $3+1$ Sterile Neutrino Scenario, JHEP 07 (2015) 171 [arXiv:1505.00978] [INSPIRE].

[101] M. Mitra, G. Senjanović and F. Vissani, Neutrinoless Double Beta Decay and Heavy Sterile Neutrinos, Nucl. Phys. B 856 (2012) 26 [arXiv:1108.0004] [INSPIRE].

[102] Planck collaboration, Planck 2018 results. VI. Cosmological parameters, Astron. Astrophys. 641 (2020) A6 [Erratum ibid. 652 (2021) C4] [arXiv:1807.06209] [INSPIRE].

[103] GERDA collaboration, Probing Majorana neutrinos with double- $\beta$ decay, Science $\mathbf{3 6 5}$ (2019) 1445 [arXiv: 1909.02726] [INSPIRE]. 
[104] A. Meroni, S.T. Petcov and F. Simkovic, Multiple CP non-conserving mechanisms of $(\beta \beta)_{0 \nu}$-decay and nuclei with largely different nuclear matrix elements, JHEP 02 (2013) 025 [arXiv:1212.1331] [INSPIRE].

[105] S. Kovalenko, Z. Lu and I. Schmidt, Lepton Number Violating Processes Mediated by Majorana Neutrinos at Hadron Colliders, Phys. Rev. D 80 (2009) 073014

[arXiv: 0907.2533] [INSPIRE].

[106] CUORE collaboration, First Results from CUORE: A Search for Lepton Number Violation via $0 \nu \beta \beta$ Decay of ${ }^{130}$ Te, Phys. Rev. Lett. 120 (2018) 132501 [arXiv:1710.07988] [INSPIRE].

[107] GERDA collaboration, Improved Limit on Neutrinoless Double- $\beta$ Decay of ${ }^{76}$ Ge from GERDA Phase II, Phys. Rev. Lett. 120 (2018) 132503 [arXiv:1803.11100] [InSPIRE].

[108] KamLAND-Zen collaboration, Search for Majorana Neutrinos near the Inverted Mass Hierarchy Region with KamLAND-Zen, Phys. Rev. Lett. 117 (2016) 082503 [Addendum ibid. 117 (2016) 109903] [arXiv: 1605. 02889] [INSPIRE].

[109] J.T. Penedo and S.T. Petcov, The $10^{-3}$ eV frontier in neutrinoless double beta decay, Phys. Lett. B 786 (2018) 410 [arXiv:1806.03203] [inSPIRE].

[110] J. Kopp, P.A.N. Machado, M. Maltoni and T. Schwetz, Sterile Neutrino Oscillations: The Global Picture, JHEP 05 (2013) 050 [arXiv: 1303.3011] [INSPIRE]. 